\title{
3D mapping of the dense interstellar gas around the Local Bubble ${ }^{\star}$
}

\author{
R. Lallement ${ }^{1}$, B. Y. Welsh ${ }^{2}$, J. L. Vergely ${ }^{3}$, F. Crifo ${ }^{4}$, and D. Sfeir ${ }^{2}$ \\ 1 Service d'Aéronomie du CNRS, 91371 Verrières-le-Buisson, France \\ 2 Experimental Astrophysics Group, Space Sciences Laboratory, UC Berkeley, CA 94720, USA \\ 3 ACRI-ST, BP 234, 06504 Sofia-Antipolis, France \\ ${ }^{4}$ GEPI and URA 8111 du CNRS, Observatoire de Paris, 92195 Meudon, France
}

Received 21 February 2003 / Accepted 30 July 2003

\begin{abstract}
We present intermediate results from a long-term program of mapping the neutral absorption characteristics of the local interstellar medium, motivated by the availability of accurate and consistent parallaxes from the Hipparcos satellite. Equivalent widths of the interstellar NaI D-line doublet at $5890 \AA$ are presented for the lines-of-sight towards some 311 new target stars lying within $\sim 350 \mathrm{pc}$ of the Sun. Using these data, together with $\mathrm{NaI}$ absorption measurements towards a further $\sim 240$ nearby targets published in the literature (for many of them, in the directions of molecular clouds), and the $\sim 450$ linesof-sight already presented by (Sfeir et al. 1999), we show 3D absorption maps of the local distribution of neutral gas towards 1005 sight-lines with Hipparcos distances as viewed from a variety of different galactic projections.

The data are synthesized by means of two complementary methods, (i) by mapping of iso-equivalent width contours, and (ii) by density distribution calculation from the inversion of column-densities, a method devised by Vergely et al. (2001). Our present data confirms the view that the local cavity is deficient in cold and neutral interstellar gas. The closest dense and cold gas "wall", in the first quadrant, is at $~ 55-60$ pc. There are a few isolated clouds at closer distance, if the detected absorption is not produced by circumstellar material.

The maps reveal narrow or wide "interstellar tunnels" which connect the Local Bubble to surrounding cavities, as predicted by the model of Cox \& Smith (1974). In particular, one of these tunnels, defined by stars at 300 to 600 pc from the Sun showing negligible sodium absorption, connects the well known CMa void (Gry et al. 1985), which is part of the Local Bubble, with the supershell GSH 238+00+09 (Heiles 1998). High latitude lines-of-sight with the smallest absorption are found in two "chimneys", whose directions are perpendicular to the Gould belt plane. The maps show that the Local Bubble is "squeezed" by surrounding shells in a complicated pattern and suggest that its pressure is smaller than in those expanding regions.

We discuss the locations of several $\mathrm{HI}$ and molecular clouds. Using comparisons between $\mathrm{NaI}$ and $\mathrm{HI}$ or $\mathrm{CO}$ velocities, in some cases we are able to improve the constraints on their distances. According to the velocity criteria, MBM 33-37, MBM 16-18, UT 3-7, and MBM 54-55 are closer than $\sim 100$ pc, and MBM 40 is closer than 80 pc. Dense HI clouds are seen at less than 90 pc and $85 \mathrm{pc}$ in the directions of the MBM 12 and MBM 41-43 clouds respectively, but the molecular clouds themselves may be far beyond. The above closest molecular clouds are located at the neutral boundary of the Bubble. Only one translucent cloud, G192-67, is clearly embedded within the LB and well isolated.

These maps of the distribution of local neutral interstellar NaI gas are also briefly compared with the distribution of both interstellar dust and neutral HI gas within 300 pc.
\end{abstract}

Key words. Galaxy: solar neighborhood - ISM: atoms - ISM: clouds

\section{Introduction}

Our unique placement within a galactic region of very low interstellar neutral gas density (i.e. the "Local Bubble" cavity, hereafter LB) enables us to directly measure the physical state of the local interstellar gas through accurate absorption

Send offprint requests to: $\mathrm{R}$. Lallement, e-mail: rosine.lallement@aerov.jussieu.fr

* Tables 1 and 2 are only available in electronic form at the CDS via anonymous ftp to cdsarc.u-strasbg.fr (130.79.128.5) or via http:cdsweb.u-strasbg.fr/cgi-bin/qcat?J/A+A/411/447 measurements that are essentially uncontaminated by intervening line-of-sight interstellar features. The spatial structure of the neutral absorption characteristics of gas within the local interstellar medium (LISM) has recently been revealed by two surveys of $\mathrm{NaI}$ ( $\lambda 5890 \AA$ ) absorption measured along 293 (Welsh et al. 1994) and 456 (Sfeir et al. 1999, hereafer Paper I) lines-of-sight within $300 \mathrm{pc}$ of the Sun. Those NaI data, as well as HI data listed by Diplas \& Savage (1994) and Fruscione et al. (1994) have also been converted into 3D density distribution by Vergely et al. (2001). 
Absorption due to the NaI ion is a good indicator of the total amount of neutral interstellar gas in a particular sightline, since NaI generally resides in "cold" $(T<1000 \mathrm{~K})$ and predominantly neutral interstellar regions (Hobbs 1978, but note comments by Welty et al. 1994). Previous studies have revealed a paucity of $\mathrm{NaI}$ absorption associated with warm and diffuse clouds within the Local Bubble region. In particular, in the case of the group of clouds surrounding the Sun the measured $\mathrm{NaI}$ equivalent widths do not exceed a few $\mathrm{m} \AA$ (Lallement \& Ferlet 1997). NaI optical data are much easier to obtain than UV data, and column-densities are much easier to extract from the HI spectra (weaker lines, and no astrospheric nor heliospheric hot gas contamination). The NaI observations have revealed an absorption threshold, which has been interpretated as a "wall" for the cold, relatively dense neutral gas with an equivalent hydrogen column density, $N(\mathrm{HI}) \sim$ $3 \times 10^{19} \mathrm{~cm}^{-2}$ surrounding the LB cavity with radii varying between 65 to $150 \mathrm{pc}$. These findings are supported in three ways by (i) the very low levels found locally in the $3 \mathrm{D}$ density distribution maps of HI which is qualitatively correlated with that of NaI (Vergerly et al. 2001), (ii) the very low values of interstellar reddening found towards stars within $50 \mathrm{pc}$ (Knude \& Høg 1998), and (iii) the galactic distribution of extreme ultraviolet sources which are mostly contained within the neutral boundary of the local cavity as derived from both interstellar NaI and HI absorption studies (Welsh et al. 1999).

The existence of this threshold in distance does not necessarily mean that there is a real "wall" of gas in all directions, and this is still debated (Mebold et al. 1998), as well as the origin of the Local Bubble cavity. For a review on the LB formation "scenarios", see Cox (1998). It is widely believed that the cavity is mainly filled with rarefied and hot $\left(10^{6} \mathrm{~K}\right)$ gas, an assumption based largely upon the interpretation of observations of the distribution of the soft X-ray diffuse background emission (Snowden et al. 1998). The local search for highly ionized gas at hotter temperatures has remained elusive. For example, preliminary observations by the NASA Far Ultraviolet Spectroscopic Explorer (FUSE) towards several local $(d<70 \mathrm{pc})$ hot white dwarf stars have failed to detect OVI ( $\lambda 1032 \AA$ ) absorption (indicative of $300000 \mathrm{~K}$ gas) to a level of $\mathrm{N}(\mathrm{OVI})<5 \times 10^{12} \mathrm{~cm}^{-2}$ (Oegerle et al. 2000). In addition, absorption measurements using both the Hubble Space Telescope (HST) Goddard High resolution Spectrometer (GHRS) and Imaging Spectrograph (STIS) towards nearby hot stars and white dwarfs have also failed to detect appreciable levels of high ionization CIV and SiIV gas $(T \sim 80000 \mathrm{~K})$ for distances <100 pc (Bertin et al. 1995; Holberg et al. 1999). However, all 3 of these high ionization line species have been routinely detected with significant column densities by the FUSE and HST instruments for sight-lines with distances $>150 \mathrm{pc}$, i.e. beyond the nominal LB neutral boundary. We note that most theoretical models of the LB favor the production of high ions in collisional ionization equilibrium at a conductive interstellar cloud interface (Slavin 1989). Because many small, low-density partially ionized ("local fluff") gas clouds have been revealed by high or ultra-high spectral resolution observations of the interstellar CaII K-line towards stars within 30 pc (e.g. Lallement et al. 1986; Vallerga et al. 1993;
Welty et al. 1986; Crawford 2001), one expects a significant number of such interfaces along any longer line-of-sight, in contrast to the absence of CIV, SiIV and OVI quoted above.

Thus, with the exception of the line-of-sight towards the bright Extreme UltraViolet (EUV) star $\epsilon$ CMa (Gry \& Jenkins 2001) which shows CIV at the radial velocity of the local cloud, but possibly tracing more distant gas having by coincidence the same radial velocity, the presently available data seems to favor a LB filled with very low density highly ionized (high temperature) gas, with possibly a production site for such ions at the interface between the LB and the cold clouds at its boundary, but very low production of ions at the interfaces of clouds embedded in the bubble. We note that a radically different theory of the LB that includes adiabatic cooling predicts temperatures as low as $50000 \mathrm{~K}$ for the local non-collisionally ionized gas, but the recent model predictions by Breitschwerdt (2001) for CIII (977 $\AA$ ) emission in the LB are contrary to the very low levels recently observed by FUSE (Welsh et al. 2002).

There are several other "anomalies" about the hot and the warm gas in the LB. The gas pressure in the Local Cloud surrounding the Sun is now precisely known from measurements in the heliosphere, and, with $P / k=2200 \pm 500 \mathrm{~cm}^{-3} \mathrm{~K}$ (Lallement 1997), is between 5 and 10 times less than the pressure deduced from the soft X-ray background. This is in agreement with the low pressure also found in other clouds embedded in the LB by Jenkins (2002). The way magnetic pressure can help to fill the gap is still unclear, and in the case of the local cloud, hardly compatible with the observations. There are also several difficulties with ionization states: helium is found to be $35-40 \%$ ionized in all diffuse clouds within 30 pc (Wolff et al. 1999). This ionization state can be explained by line emission from conductive interfaces between clouds and hot gas (Slavin 1989; Jenkins et al. 2000), but we have discussed above the absence of ions in these interfaces. On the other hand, Jenkins et al. (2000) also discuss the relative ionizations of $\mathrm{Ar}, \mathrm{Ne}, \mathrm{N}$, and $\mathrm{H}$, which according to those authors imply a strong photoionizing field by stars and hot gases.

Finally we note that charge transfer between solar wind ions and neutral interstellar gas in the heliosphere may contribute significantly to the soft X-ray background (Cox 1998; Cravens 2000). Recent model calculations by Robertson et al. (2001) have shown that this heliospheric emission can account for up to $50 \%$ of the observed soft X-ray background intensity, and thus there is a growing controversy as to whether the LB contains any million degree $\mathrm{K}$ gas at all! We note that over the next few years the spatial characteristics of local $(d<500 \mathrm{pc})$ hot $(T=300000 \mathrm{~K})$ gas in absorption will be mapped by the FUSE satellite using the OVI ion, and the local hot $(T=500000$ to 1 million degree $\mathrm{K}$ ) gas in emission will be surveyed by the NASA Cosmic Hot Interstellar Plasma Explorer (CHIPS) (Hurwitz \& Sholl 1999). A key piece of information that both surveys will require in order to correctly interpret their respective data is an accurate 3D galactic map of the neutral boundary to the LB cavity. As mentioned previously, a preliminary absorption map has already been presented in Paper I, but only $456 \mathrm{NaI}$ absorption sight lines were sampled, with the majority of observations having been taken from northern hemisphere observatories. In this paper we present a 
further 311 line-of-sight measurements using high spectral resolution $\mathrm{NaI}$ absorption data collected at ground-based observatories in both hemispheres, together with a further 239 measurements published in the literature since Paper I. These new maps, based on a grand total of 1005 sight-lines, support many of the findings of the preliminary absorption survey presented in Paper I and confirm the placement of the neutral boundary to the LB to within an accuracy of $\sim \pm 20 \mathrm{pc}$ in many directions. In addition we compare our new maps of the LB with the local distribution of interstellar dust and $\mathrm{HI}$ gas, and we also discuss the position and possible physical relationship between the local cavity and the nearest HI supershells. Finally, we discuss the location of nearby molecular clouds and their placements with respect to the local cavity. This can help in the interpretation of the shadows in the soft X-ray background emission.

\section{Observations and data reduction}

Observations of both the interstellar NaI D1 and D2-lines at $\sim 5890 \AA$ were obtained during 11 observing runs over the period 1998-2000 using the following instrumentation: (i) March 1998, September 1998, January 1999 and April 2000 using the Aurelie spectrograph at the $1.52 \mathrm{~m}$ telescope of the Observatoire de Haute Provence (France), (ii) June 1998 and 1999 using the Hamilton echelle spectrograph on the $0.9 \mathrm{~m}$ coudé feed telescope at the Lick Observatory (USA), (iii) March 2000 using the echelle spectrograph on the Kitt Peak 0.9 m coudé feed telescope, (iv) April 1998, December 1999 and August 2000 using the coudé echelle spectrograph on the $1.4 \mathrm{~m}$ and $3.6 \mathrm{~m}$ telescopes at the European Southern Observatory (La Silla, Chile), and (v) December 1999 using the coude echelle spectrograph at the 74 inch telescope of the Mt. Stromlo Observatory (Canberra, Australia).

The new sight-lines were sampled using the criteria that target stars possess: (i) a Hipparcos catalog distance (ESA 1997) and an associated relative standard error (see Paper I) smaller than 0.3 , resulting in distances $<350 \mathrm{pc}$, (ii) a galactic position that filled distance gaps in the data of Paper I, (iii) a spectral type earlier than $\mathrm{A} 5 \mathrm{~V}$, and (iv) a stellar rotational velocity $>30 \mathrm{~km} \mathrm{~s}^{-1}$. In some special cases we have relaxed these criteria in order to observe a small number of more distant stars (generally $<500 \mathrm{pc}$ ) in order to better define and constrain the position of the LB boundary. In Table 1, available at the CDS, we list the sight-lines to the 311 newly observed target stars and give values for their galactic longitude and latitude, spectral type, visual magnitude and Hipparcos distance (in pc). These data were compiled using the SIMBAD data retrieval system of the Astronomical Data center in Strasbourg (France) and the on-line Hipparcos catalog (ESA 1997). The spectral resolution of the interstellar observations taken at each Observatory was checked against that of a Th-Ar calibration lamp, resulting in a spectral resolution of $3 \mathrm{~km} \mathrm{~s}^{-1}$ for the OHP data, $5.5 \mathrm{~km} \mathrm{~s}^{-1}$ for the Lick Observatory data, $3.2 \mathrm{~km} \mathrm{~s}^{-1}$ for the Kitt Peak data, $3.0 \mathrm{~km} \mathrm{~s}^{-1}$ for the ESO data and $5.0 \mathrm{~km} \mathrm{~s}^{-1}$ for the Mt. Stromlo data.

All the data were reduced in an identical manner to that outlined in Paper I. Briefly this involved division by a flatfield and instrumental background light subtraction, removal of telluric water vapor lines using a synthetic transmission spectrum and the establishment of a wavelength scale from those telluric lines. In general, the accuracy of the wavelength calibration using the telluric lines was $\pm 1 \mathrm{~km} \mathrm{~s}^{-1}$. Our telluric line removal process has been described in detail in Lallement et al. (1993) and for a typical observation this results in an absorption residual of less than $1 \mathrm{~m} \AA$ at the location of the $\mathrm{D} 2$ line.

The resultant spectra were then fitted with a high order polynomial to establish a local stellar continuum level, such that the equivalent widths of the D2 and D1 lines could be measured. For most of our targets (which are mainly fast rotating O, B and A stars) the stellar continuum is essentially flat and easy to establish (see Fig. 2). For the majority of our targets the $\mathrm{NaD}$ lines are unsaturated. The measured values of equivalent widths for the D2 and D1 lines to all our targets are listed in Table 1. All the spectra were well exposed, such that for a typical observation a minimum $\mathrm{S} / \mathrm{N}$ ratio of 30:1 was obtained which resulted in a D2-line equivalent width detection limit of $<3.0 \mathrm{~m} \AA$ for most of the observations. Typically, an error of $\sim 10 \%$ can be assigned to the measurement of an equivalent width value given in Table 1 . There are several cases where our target stars have been previously observed by other authors and in general our equivalent width measurements agree to within $\pm 10 \%$. In Fig. 2 we show some typical examples of our spectral data. In these figures we also show the raw spectrum prior to telluric line removal, the placement of the stellar continuum level and the final processed residual intensity.

A subsequent publication will report on the individual gas cloud component column densities, $N(\mathrm{NaI})$, and the corresponding velocities obtained from the fitting of the interstellar NaI absorption line profiles for all of the new sight-lines reported in both Paper I and this paper. The respective interstellar NaI residual intensity profiles for all these target stars will also be shown together with an interpretation of the global velocity field in the local ISM.

\section{Additional data sets}

Following the example set in Paper I, we have maximized the number of line-of-sight measurements of NaI in the local ISM by adding the following $\mathrm{NaI}$ absorption data to our present sample of 311 targets: (a) we have included the NaI D2-line equivalent widths for 455 sight lines (the 456 stars listed in Paper I, except one introduced by error) (b) we have added the equivalent width data listed in Table 2, available at the CDS, which is a compilation of high resolution NaI D2-line absorption measurements towards stars that have been published in the literature subsequent to those presented in Paper I, and (c) we have added $\mathrm{NaI}$ measurements to stars that were reported in papers published prior to Paper I that we unfortunately previously overlooked in the literature. Those measurements are also listed in Table 2. Our selection of these new lines-of-sight in Table 2 is generally restricted to those stars with Hipparcos distances $<350 \mathrm{pc}$, except in some directions where more distant targets were required to better define the LB boundary. Using these data together with our newly reported $\mathrm{NaI}$ observations of this Paper, our total of lines-of-sight (LOS) sampled is 1005. In Fig. 1 we show the galactic distribution of all these stellar 


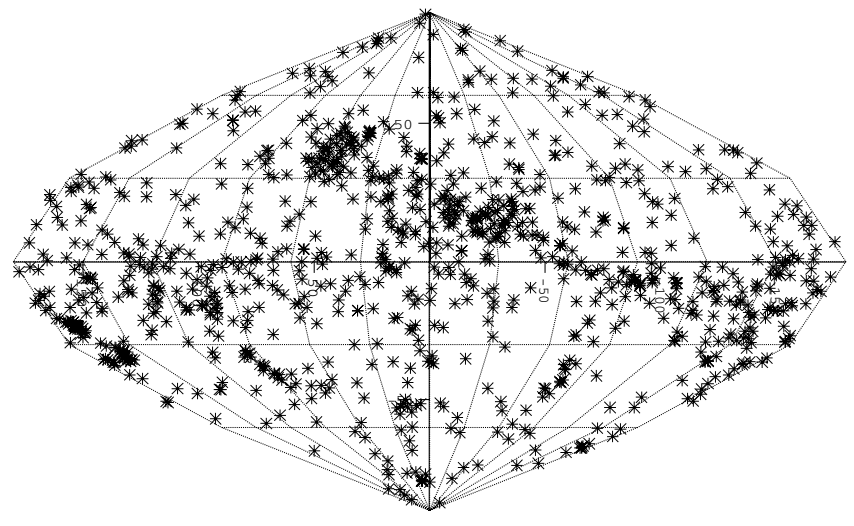

Fig. 1. The galactic distribution of all 1005 sight-lines sampled using absorption measurement of the interstellar NaI D-lines. Longitude is increasing from -180 to $180^{\circ}$ from right to left. The Gould belt, is clearly visible, because it provides the largest number of bright earlytype stars. Oversampled regions correspond to data from the literature, i.e. extensive studies of specific objects, essentially nearby molecular clouds.

sources, showing that our sky coverage is adequate in most directions except for a few small regions mainly located in the southern galactic hemisphere. It is important to note that many of the data from the literature are concentrated in specific directions, because they were targeted to probe distant molecular or HI clouds or shells, using as starting data infrared (IR) or radio maps. This has the effect of altering the otherwise random directional sampling. However, we found it important to include them because by definition they increase the number of LOS which intersect dense gas. As a matter of fact, a criticism about our previous contour study and random selection of targets is the possibility of missing nearby clumps or distant clouds characterized by small angular sizes. By doubling the total number of LOS and adding those specific targets towards dense gas, we obtain an interesting comparison with the contours of Paper I.

The existence of a dense, neutral "wall" of interstellar gas surrounding the LB was established in Paper I, in which the equivalent width of the $\mathrm{NaI}$ D2-line was observed to increase dramatically to $>20 \mathrm{~m} \AA$ over a small distance range $(60-80 \mathrm{pc})$ in the majority of galactic directions sampled (with notable exceptions such as the direction towards the $\operatorname{star} \beta \mathrm{CMa}$ ). In Fig. 3 we present a similar plot using all 1005 measurements of the interstellar NaI D2-line equivalent widths now available to us. It is clear that at distances between $70-90 \mathrm{pc}$ in the majority of galactic directions sampled the level of $\mathrm{NaI}$ absorption increases markedly from a level of $<5 \mathrm{~m} \AA$ to $>30 \mathrm{~m} \AA$. This "pileup" of interstellar gas over a relatively short distance is interpreted as being due to the presence of the neutral gas boundary to the local cavity.

In Fig. 3 we also show two best-fit lines to the data. The line with the least slope is derived for D2-line equivalent widths taken from targets with distances $<70 \mathrm{pc}$, whereas the best-fit line with the greater slope is derived from targets with distances between 70-350 pc. The large difference in slope derived from both data sets again supports the interpretation of a neutral-free interstellar gas cavity for $d<70 \mathrm{pc}$ in most directions lying close to the galactic plane.

\section{Data synthesis and representation}

\subsection{Contours of equal equivalent width}

Due to the still limited number of sight-lines, there is no straightforward method to present the data in terms of a 3D galactic distribution. In Paper I we chose a simple method, i.e. to draw contour lines of equal absorption equivalent width in selected planes. Details about the method can be found in Paper I. Here we use a similar method. Basically, we select all stars located between two parallel planes distant by the "slab" thickness $e$, and we project them onto the median plane. We then compute the iso-contours as if those stars were located exactly in this plane. In order to take into account the decrease with distance of the volumic density of targets, beyond a given distance $d$ from the Sun we replace the previous criterion with the condition that the angle between the target direction and the midplane is smaller than a given angle. Here we select stars within $25 \mathrm{pc}$ from the midplane for stars closer than $80 \mathrm{pc}$, and stars with LOS at less than $17^{\circ}$ from the midplane for stars more distant than $80 \mathrm{pc}$. (Note that, in the case of the galactic plane, contours can be drawn for more restrictive conditions, because the number of targets is large enough. However, we have chosen to keep the same criteria for all the planes.) The shortcoming of such a method is that if two stars are located differently with respect to the plane (e.g. one below, one above) and they have very different absorbing columns, the isocontours will be complicated, and ultimately become inaccurate. On the other hand, since all targets are taken into account any high value of column density will not be missed. In general, this method is appropriate for detection of the threshold described in Fig. 3, i.e. for the internal contours. The distance uncertainty in the determination of the iso-contours arises from two sources: (i) the uncertainty in the Hipparcos stellar distance and (ii) the mean distance between target stars in a particular galactic direction. In general, when the iso-contour to the local cavity boundary is at a distance of less than $150 \mathrm{pc}$, the latter error source is dominant, and is $\sim \pm 20$ pc. At larger distances, (where our stellar sampling is generally less well defined), this placement error is increasing.

In the following section we show several different views of the neutral absorption characteristics of the local interstellar gas $(d<300 \mathrm{pc})$ using contours of the NaI D2-line absorption strength plotted as a function of the stellar galactic coordinates projected onto different galactic planes of reference. Due to the larger number of $\mathrm{NaI}$ lines-of-sight now available we are able to substantially improve the spatial coverage of the 3D maps originally shown in Paper I and increase the accuracy of the contour plot.

In the following figures we have constructed NaI D2-line equivalent width absorption contours at intervals of $20 \mathrm{~m} \AA$ and $>50 \mathrm{~mA}$. It has been shown that $N(\mathrm{NaI})$ is well correlated with the total column density of neutral hydrogen, $N(\mathrm{HI})$, for values of $\log N(\mathrm{NaI}) 11.0-13.0 \mathrm{~cm}^{-2}$, although for smaller values of $N(\mathrm{NaI})$ this correlation can have large errors (Welty et al. 1994; Ferlet et al. 1985). As a guide, a NaI D-2 equivalent width of $20 \mathrm{~m} \AA$ corresponds approximately to a neutral hydrogen column density of $\log N(\mathrm{HI})=19.3 \mathrm{~cm}^{-2}$, and that of a 

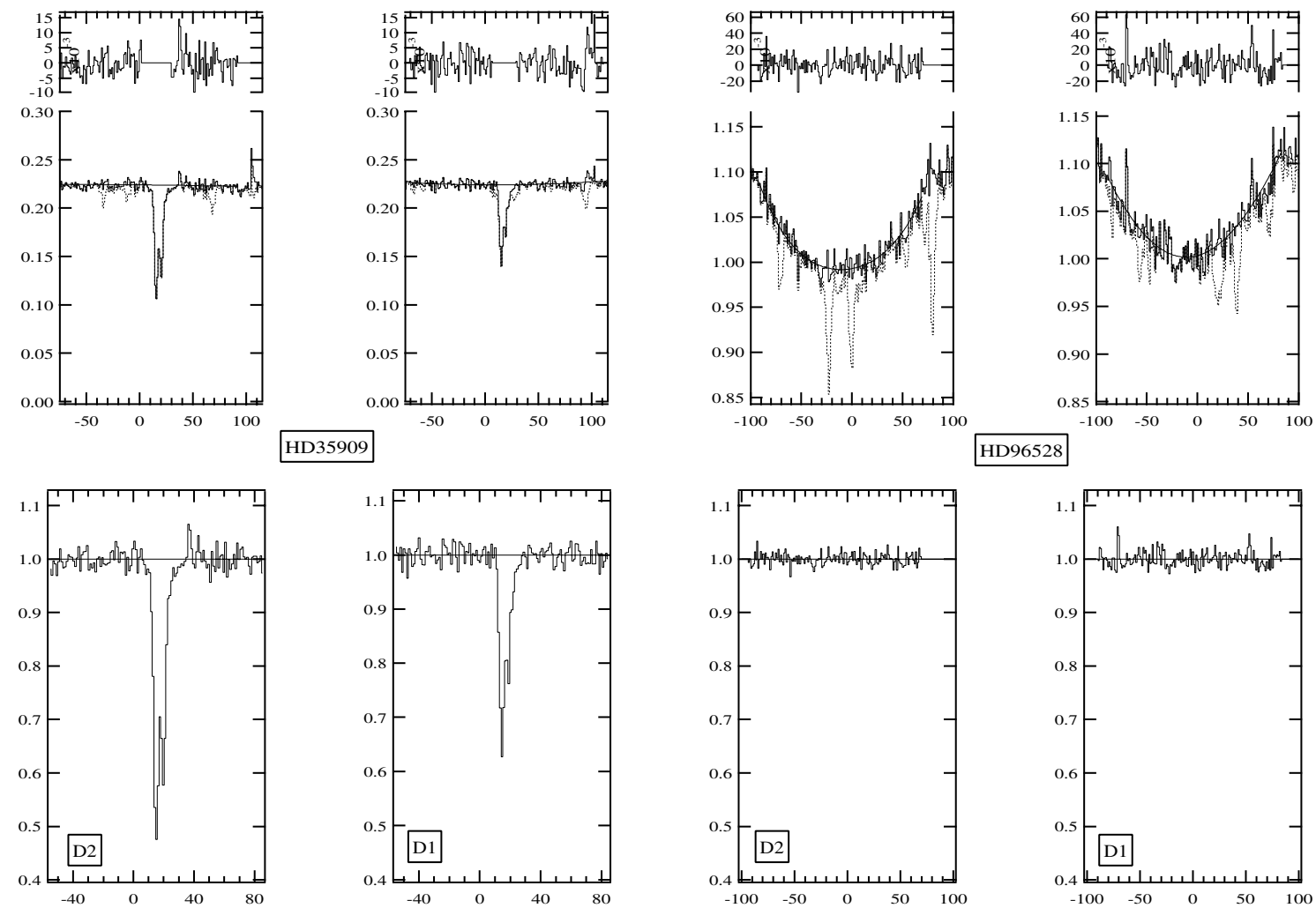

Fig. 2. Examples of NaI D2 and D1 spectral regions, water vapor removal and stellar continuum fitting: (i) a hot star with a flat continuum and a small number of sharp interstellar lines due to intervening gas in cold clouds. This case is representative of most of our target stars located beyond the first dense clouds defining the LB boundary (left). (ii) a cooler star and the absence of sharp interstellar lines (right). After telluric water vapor line removal and continuum fitting by a fourth order polynomial, an upper limit for the NaI-D2 equivalent width is easily derived.

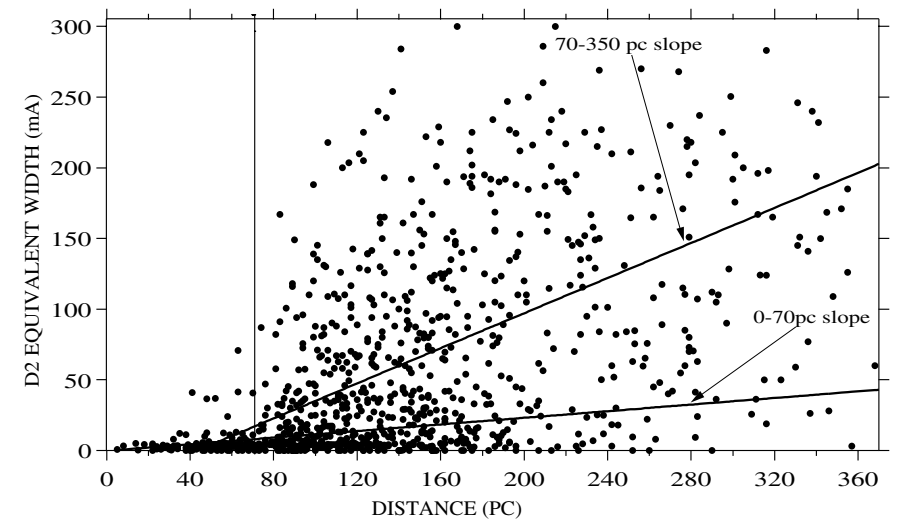

Fig. 3. Plot of the Na D-2 equivalent width (mÅ) versus distance (pc) to all 1005 sight-lines. Best linear fits to the data are shown for sightlines of distance 0 to $70 \mathrm{pc}$ and for 70 to $350 \mathrm{pc}$.

$50 \mathrm{~m} \AA$ line corresponds to $\log N(\mathrm{HI})>20 \mathrm{~cm}^{-2}$. We identify sight-lines with column densities in excess of this latter value as containing sufficient neutral gas to define a plausible absorption boundary to the rarefied LB cavity.

It is also noteworthy that in the following three detailed plots of the shape of the LB in the galactic plane (Fig. 4), the meridian plane (Fig. 5) and the rotational plane (Fig. 6), the distance over which the equivalent width of $\mathrm{NaI}$ changes from $<20 \mathrm{~m} \AA$ to $>50 \mathrm{~m} \AA$ is typically only $\sim 30 \mathrm{pc}$. This is merely a pictorial representation of the results shown in Fig. 3 that confirms the existence of a marked build-up of neutral density interstellar gas, which we associate with the LB boundary. Note that in order to help the reader assess the accuracy of the isocontour placement in a galactic direction, we have included the positions of all our stellar targets on each of Figs. 4 to 6 .

\subsection{Density distribution from column-density inversion}

We have applied the robust inversion method of Vergely et al. (2001) to our NaI data set in order to derive volumic densities in $3 \mathrm{D}$ space using integrated column densities derived from our measured equivalent widths. For the majority of our targets the $\mathrm{Na}$ D-lines are unsaturated and column density values can be derived with an accuracy of $\sim \pm 20 \%$. For strong absorptions we have converted the equivalent widths into column-densities by means of an empirical relationship established in Paper I. The choice of this relationship does not influence the resulting shape of the cavity, it only slightly alters the density gradients within a region of very high local gas density.

Although full details of the inversion method can be found in Vergely (2001), we briefly recall here the basic principles. The computation starts with an a priori model for the density distribution, here an exponential decrease with height above the galactic plane

$\rho_{\text {prior }}(x, y, z)=\rho_{0} \exp \left(-|z| / h_{0}\right)$.

The inverse process calculates the fluctuations produced by the lines-of-sight constraints around this initial model. The space 


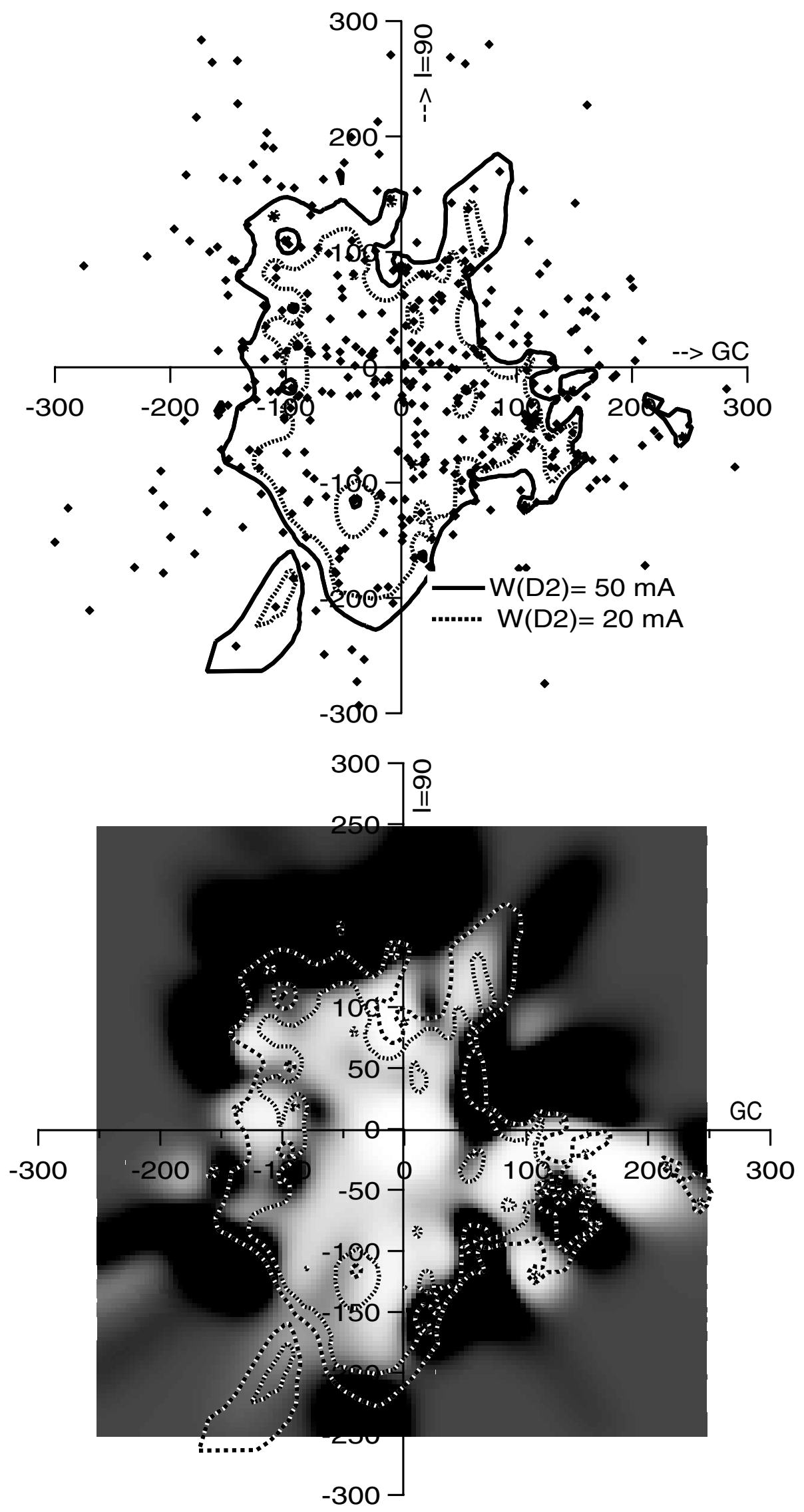

Fig. 4. Dense gas along the galactic plane: iso equivalent width contours for $W=20 \mathrm{~m} \AA$ and $50 \mathrm{~m} \AA$ resp. (top), calculated from 426 selected stars, and cut in the 3D volumic density obtained from the global inversion of the column-densities (bottom). 

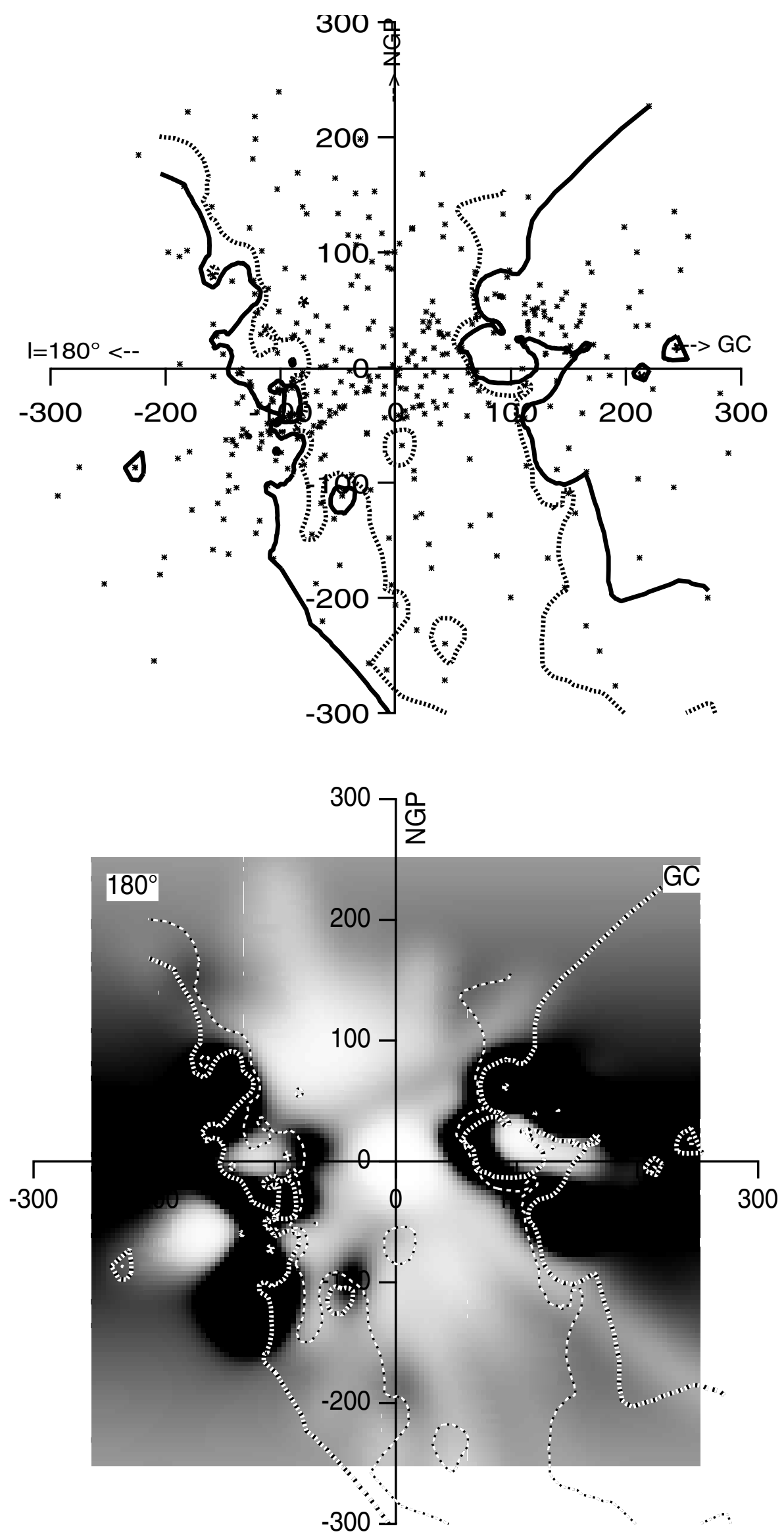

Fig. 5. Same as Fig. 4, in the meridian plane. The iso-contours are derived from 387 stars. 

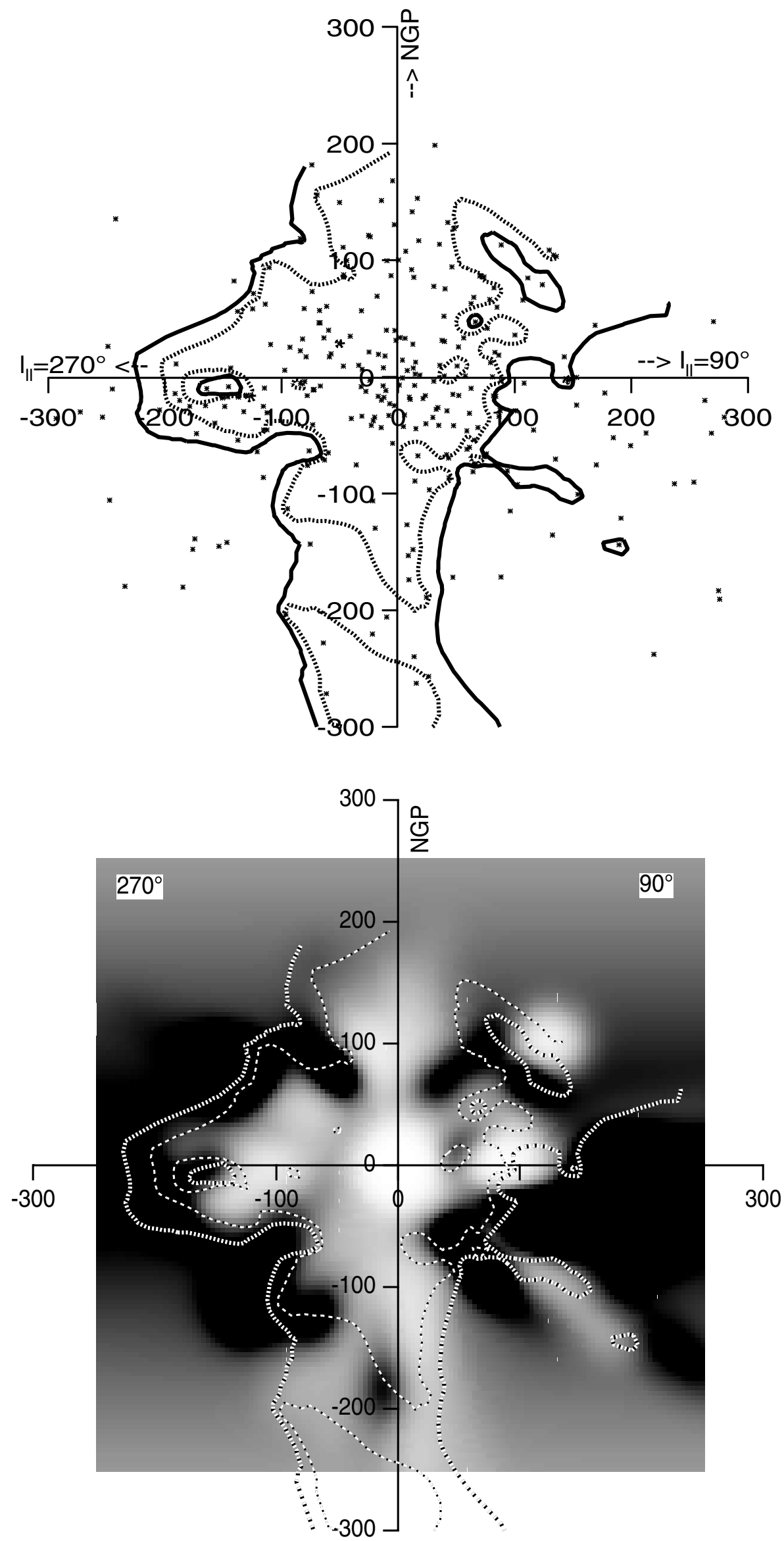

Fig. 6. Same as Fig. 4, in the rotation plane. The iso-contours are derived from 283 stars. 
density fluctuation at $x, y, z$ is parameterized by the lognormal law:

$\alpha(x, y, z)=\log \left(\frac{\rho(x, y, z)}{\rho_{\text {prior }}(x, y, z)}\right)$.

The calculation requires strong regularizing constraints, here the interdependence between neighbouring data points, through a variance-covariance operator. The interdependence is controlled by the two parameters $\xi$, the smoothing length, and $\sigma_{\alpha}$, the fluctuation level, and the condition that for each pair of space points the autocorrelation function must be equal to the following kernel of a variance-covariance operator (Tarantola \& Valette 1982):

$$
\begin{aligned}
& C_{0}\left(x, x^{\prime}, y, y^{\prime}, z, z^{\prime}\right)=\sigma_{\alpha}(x, y, z) \sigma_{\alpha}\left(x^{\prime}, y^{\prime}, z^{\prime}\right) \\
& * \exp \left(-\frac{\left(\left(x-x^{\prime}\right)^{2}+\left(y-y^{\prime}\right)^{2}+\left(z-z^{\prime}\right)^{2}\right)}{\xi^{2}}\right) .
\end{aligned}
$$

The smoothing length $\xi$ corresponds roughly to the mean distance between two neighbouring target stars and the fluctuation level $\sigma_{\alpha}$ varies asymptotically as

$\sigma_{\alpha} \propto\left(\frac{1}{\xi}\right)^{\frac{3}{2}}$.

The fluctuations are computed according to an iterative process described in details in Vergely et al. (2001) (Appendix), which converges after about 10 iterations. A $\chi^{2}$ minimization criteria controls the algorithm convergence:

$\chi^{2}=\sum_{i=1, N} \frac{\left(N_{i(\text { obs })}-N_{i(\text { model })}\right)^{2}}{\sigma_{N_{i}}^{2}}$, where $\sigma_{N_{i}}$ is the error on the column density $N_{i}$ and the sum is on the $N$ lines-of-sight. Errors on the columns and on star distances are combined into a single parameter (see Vergely et al. 2001), which is the quadratic sum of the Hipparcos error on the parallax, the error on the column density (taken at $25 \%$ of the column), and an additional term to take into account errors on the weak columns and non detections. A specific data error modelisation has been performed with the following characteristics:

- Gaussian distribution behavior near the data measurement;

- exponential distribution behavior far from the data measurement.

It allows us to obtain reliable results in accordance with the exponential norm when the data error bars have been underestimated.

For this comparison we have only slightly changed the parameters used by Vergely et al. (2001), using $h_{0}=200 \mathrm{pc}$, and $\rho_{0}=1 \times 10^{-9} \mathrm{~cm}^{-3}$. However, we have significantly reduced the smoothing length $\xi$, from 40 to $25 \mathrm{pc}$, such that it now corresponds to the distance to the mid-plane in our isocontour plotting method. Also we note that Vergely et al. used $\mathrm{NaI}$ column densities derived from an assumed linear relationship with the measured equivalent width (which in most cases is valid). In our present paper we use the more accurate values of NaI column density derived from the empirical relationship derived in Paper I.

\subsection{Advantages and shortcomings of both methods}

The two methods are basically different, and bring complementary information. The contour method is appropriate if one wants to derive the maximum distance to a gas condensation (the maximum "empty" path), which was the primary goal of the survey, but it is simplistic. It does not reject any data point, and it has revealed errors in data interpretations, i.e. absorption initially attributed to the ISM but is actually of circumstellar or stellar origin. Such data points have been removed or, when it has been possible, the ISM columns have been corrected in the input list. The major shortcomings of the contour method is that it is not able to describe correctly the absorption characteristics beyond the closest condensation (see discussions below). In addition, the significance of the contour disappears at large distances, where stars very distant from each other are treated as being close to the same plane. At variance with this method, the inversion method is a powerful and sophisticated tool, which is fully appropriate to reveal masses of gas at any distance, provided there are constraining target measurements. On the other hand, it is somewhat dependent on the a priori distribution, and as any least mean squares method it may minimize or maximize the weight of some data points. If some density fluctuations have characteristic scales smaller than the correlation length, such fluctuations will be smoothed out or ignored because they are too far from the a priori solution.

Figures 4 to 6 clearly show those respective properties of the two methods. While in general they agree on the global shape of the local cavity, maxima in the density distribution are often slightly displaced from what the contours suggest, due to the smoothing length (in the contour method gradients can be smooth or sharp, depending on the constraints), and in some cases clumps have been minimized or associated with another neighboring mass of gas. Figures 5 and 6 also show the distribution with $z$, because where there are no constraints, at large distances along the plane, the density follows the initial density distribution.

\section{The LISM viewed from above the galactic plane - Fig. 4}

Due to the large number of sight-lines available for sampling in this particular galactic projection, the extent of the low density cavity in the plane of the galactic disk is most accurately revealed. With respect to the contour plot originally presented in Paper I, we note that the complexity of the LB contours is now better revealed. The $50 \mathrm{~m} \AA$ contour is no longer open, which means that in the selected volume of space we find more targets with high columns, which has the effect of closing the inner contour line. The automatic contour program now shows the holes towards $l=240^{\circ}$ and towards $l=330^{\circ}$ as secondary contours at larger distance. Apart from those regions, it is interesting to note that, despite the large number of targets, including those located towards high density clouds, the distance to the inner contours at 20 and $50 \mathrm{~m} \AA$ has not decreased. In particular, the distance to the dense gas towards the galactic center is defined by a large number of stars and still found to be about $60 \mathrm{pc}$. 
We also note the complete absence of isolated dense gas condensations ( $\mathrm{NaI}$ D2-line equivalent width $>50 \mathrm{~mA}$ ) within $60 \mathrm{pc}$ of the Sun in all directions.

\subsection{Condensations of gas in the cavity}

We have made a systematic search of the clumps of gas residing within the LB boundary to determine if their origin is truly interstellar, stellar or circumstellar. There is a line of dense clumps between $l=130^{\circ}$ and $l=190^{\circ}$ which are located very close to the position of the nominal $50 \mathrm{~m} \AA$ contour to the LB boundary. We believe these condensations are actually part of the boundary itself and only appear to lie within the local cavity due to the finite thickness of the projection slice used to produce this isocontour. For completeness however, we now discuss each of these condensations to individually verify their reality.

The relatively dense region of neutral gas at $\sim 100 \mathrm{pc}$ observed with a D2-line absorption level of $>60 \mathrm{~m} \AA$ towards $l=190^{\circ}$ is associated with the stars HD $35909(\mathrm{~A} 4 \mathrm{~V})$ and HD 36162 (A3V). We note that the former target is listed as a $\lambda$ Bootis-type star by Paunzen et al. (2001), and thus the observed level of NaI absorption could be due to a circumstellar shell surrounding the star. However, this spectral classification does not apply to HD 36162 and both sets of absorption lines are centered at a heliocentric velocity of $+14 \mathrm{~km} \mathrm{~s}^{-1}$, therefore supporting an interstellar origin for this small gas cloud that appears to be located just within the LB boundary. The similarly dense clump of gas in the nearby direction of $l=170^{\circ}$ at a distance of $90 \mathrm{pc}$ (and lying within the local cavity) has been detected at levels of NaI D2-line absorption $>50 \mathrm{~m} \AA$ towards both HD 34904 and HD 28459 and is definitely of interstellar origin. We note that Lilienthal \& de Boer (1991) have also detected appreciable amounts of interstellar absorption towards HD 34904 using the I.U.E. satellite. As a conclusion, those apparent "clumps" are part of the boundary to the LB.

The small condensation of gas in the direction of $l=340^{\circ}$ at a distance of only $70 \mathrm{pc}$ is associated with the well-known chemically peculiar star $\chi$ Lup and HD 129685. Three other stars in the same sight-line with similar distances (HD 158427, HD 142629 and HD 142630) all have NaI D2-line equivalent widths $<6 \mathrm{~m} \AA$ and thus it seems highly probable that the large level of $\mathrm{NaI}$ absorption measured towards both $\chi$ Lup and HD 129685 (which has an extremely high rotational velocity of $440 \mathrm{~km} \mathrm{~s}^{-1}$ ) is likely to be of circumstellar, and not interstellar, origin.

We note that the apparent condensation of gas at $127 \mathrm{pc}$ seen towards $l=250^{\circ}$ is associated with the A0V star, HD 78955. Pauzen et al. (2001) list this as a possible $\lambda$ Bootis star, and thus the large column of NaI measured in this sightline may well be of circumstellar, and not interstellar origin.

The very small condensation of gas within the LB at $\sim 120 \mathrm{pc}$ in the direction of $l=335^{\circ}$ is associated with the star $\epsilon$ Norma (B4V). This sight-line has a NaI/CaII column density ratio of 7.8 (Welsh et al. 1997), which is typical for many interstellar sight-lines. We propose that, given its proximity to the interface between the LB and Radio Loop I, this gas cloud is most probably associated with a local disruption of the neutral boundary between the two interstellar gas shells.

Finally, our new data indicate that the condensation of dense gas at $\sim 85 \mathrm{pc}$ surrounding the star HD 211211 in the direction of galactic longitude $l=95^{\circ}$ which was placed within the LB in Paper I, is now shown to define the position of the neutral boundary to the LB in that galactic direction.

Several small, lower density condensations of gas with a $\mathrm{NaI}$ absorption level of $\sim 20 \mathrm{~m} \AA$ are also seen in Fig. 4. The nearest of these to the Sun is that detected with a D2-line equivalent width of $20.2 \mathrm{~m} \AA$ in the sight-line towards $\delta$ Cyg $(d=52 \mathrm{pc})$ at $l=79^{\circ}$ by Welty et al. (1994). This patch of neutral gas is also seen at a similar heliocentric velocity of $-19 \mathrm{~km} \mathrm{~s}^{-1}$ towards the angularly nearby stars HD 192640 $(d=41 \mathrm{pc})$ and HD $193369(d=60 \mathrm{pc})$ at D2-line levels of $41 \mathrm{~m} \AA$ and $9 \mathrm{~m} \AA$ respectively. For HD 192640 the absorption may be of circumstellar origin due to the $\lambda$ Bootis nature of this nearby star (Kamp \& Paunzen 2002).

\subsection{General features of the dense LISM in the Galactic Plane}

Absorption contours in the fourth quadrant are clearly complex, suggesting that some local disruption of the neutral gas wall may have occured. This whole region contains "holes" in the LB neutral boundary, i.e. small regions that have low levels of NaI absorption measured in long sight-lines. Such holes cannot be accurately represented by our present limited method of contour plotting. Two conspicuous tunnels (towards $l=315^{\circ}$ and towards $l=335^{\circ}$, the "Lupus tunnel", Welsh et al. 1994) are clearly seen in the density plots, which connect the LB with the Loop I cavity. These holes are also seen in the vertical plots in Figs. 5 and 8. These interstellar tunnels may possibly provide the long-sought link between the Sco-Cen OB Association and the LB cavity. The region around the North Polar Spur (Radio Loop I) supershell contains many early-type stars of the Sco-Cen OB association, and Maiz-Apellaniz (2001) has recently argued that supernovae, produced by stars in the Lower Centaurus Crux sub-group, may have created the LB cavity some $6 \mathrm{Myr}$ ago. At that time there was probably a single, preexisting low density interstellar cavity that, through the interaction of these supernovae events, split into two separate entities we now call Radio Loop I and the Local Bubble. However, there is no consensus yet about such a mechanism, and other descriptions of the formation of the LB have been given, e.g. by Berghöfer \& Breischwerdt (2002). The forthcoming study of the velocity field from our absorption spectra, and of the distribution of the warm gas should help to understand the hierarchy of explosions, and establish if the local warm clouds in the solar vicinity are the precursors of the debris of the collision between two expanding shells (Egger \& Ashenbach 1995; Breitschwerdt et al. 2000).

The antigalactic center area, between 80 and $150 \mathrm{pc}$, is also complex. This region illustrates very well the complementary nature between the two methods we are using. The $>20 \mathrm{~m} \AA$ contour shows that between $l=150$ and $210^{\circ}$, the local cavity extends to $80 \mathrm{pc}$. Beyond this boundary, the method 

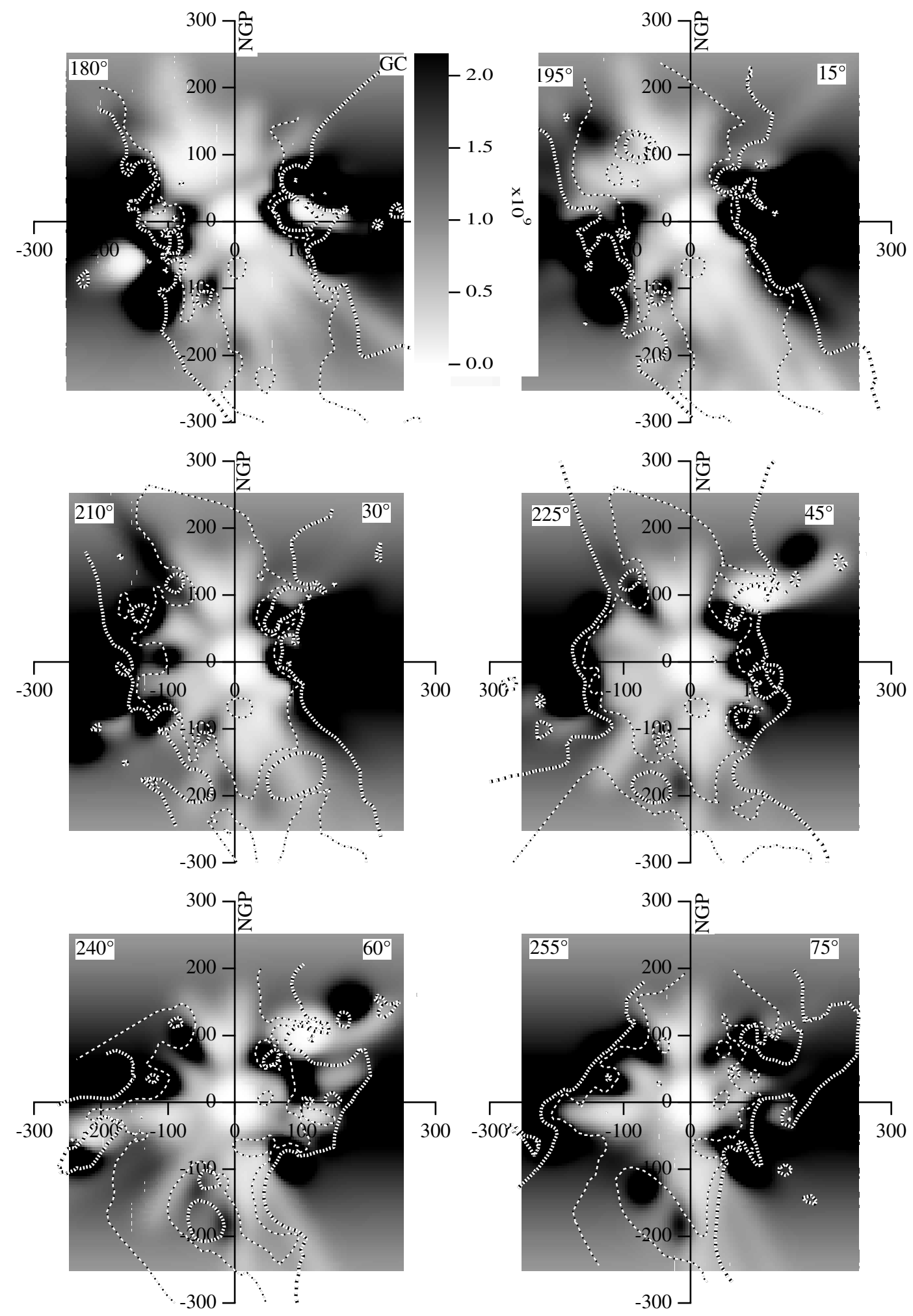

Fig. 7. Dense gas along vertical planes rotating around the polar axis plotted by 15 deg steps: iso equivalent width contours, and volumic density after inversion of the column-densities.

of automatic contours is less appropriate, as suggested by the clumps (see above), and the distance between the $>20 \mathrm{~m} \AA$ and the $>50 \mathrm{~m} \AA$ contours is more extended than in other directions. The density distribution map on the other hand reveals a cavity between 65 and $150 \mathrm{pc}$, and an HI "wall" which separates this cavity from the LB. From the combination of both methods, as discussed above, we conclude that there must be a dense wall starting at $80 \mathrm{pc}$, and beyond this wall a cavity extending up to about $150 \mathrm{pc}$. This HI "wall" has been already studied with IUE by Lilienthal \& de Boer (1991), who concluded 

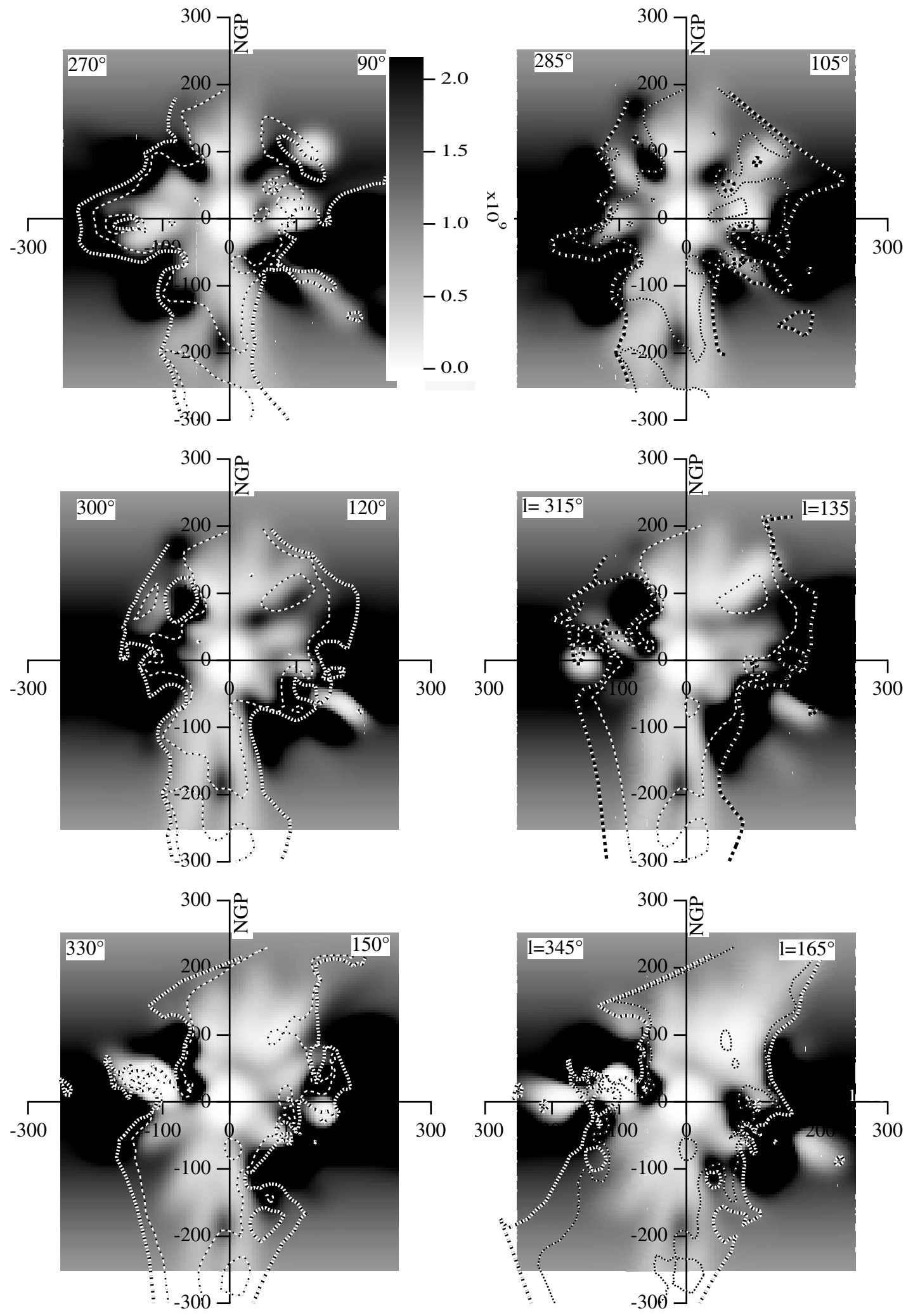

Fig. 8. Same as Fig. 7 for the next set of vertical planes.

that this dense gas is associated with the interaction region between the Auriga-Perseus complex and the boundary to the LB. Indeed, when applying Hipparcos distances to their results we find that the distance to the inner part of the shell is constrained by IUE data in the interval $65-90 \mathrm{pc}$, and that our additional
$\mathrm{NaI}$ data further reduce this interval to $75-85 \mathrm{pc}$. We identify the region devoid of gas seen beyond the HI gas as the void created by the Pleiades stellar cluster.

In the first quadrant, a smaller tunnel appears towards $l=$ $65^{\circ}$, which extends to $160 \mathrm{pc}$, while dense gas is detected much 


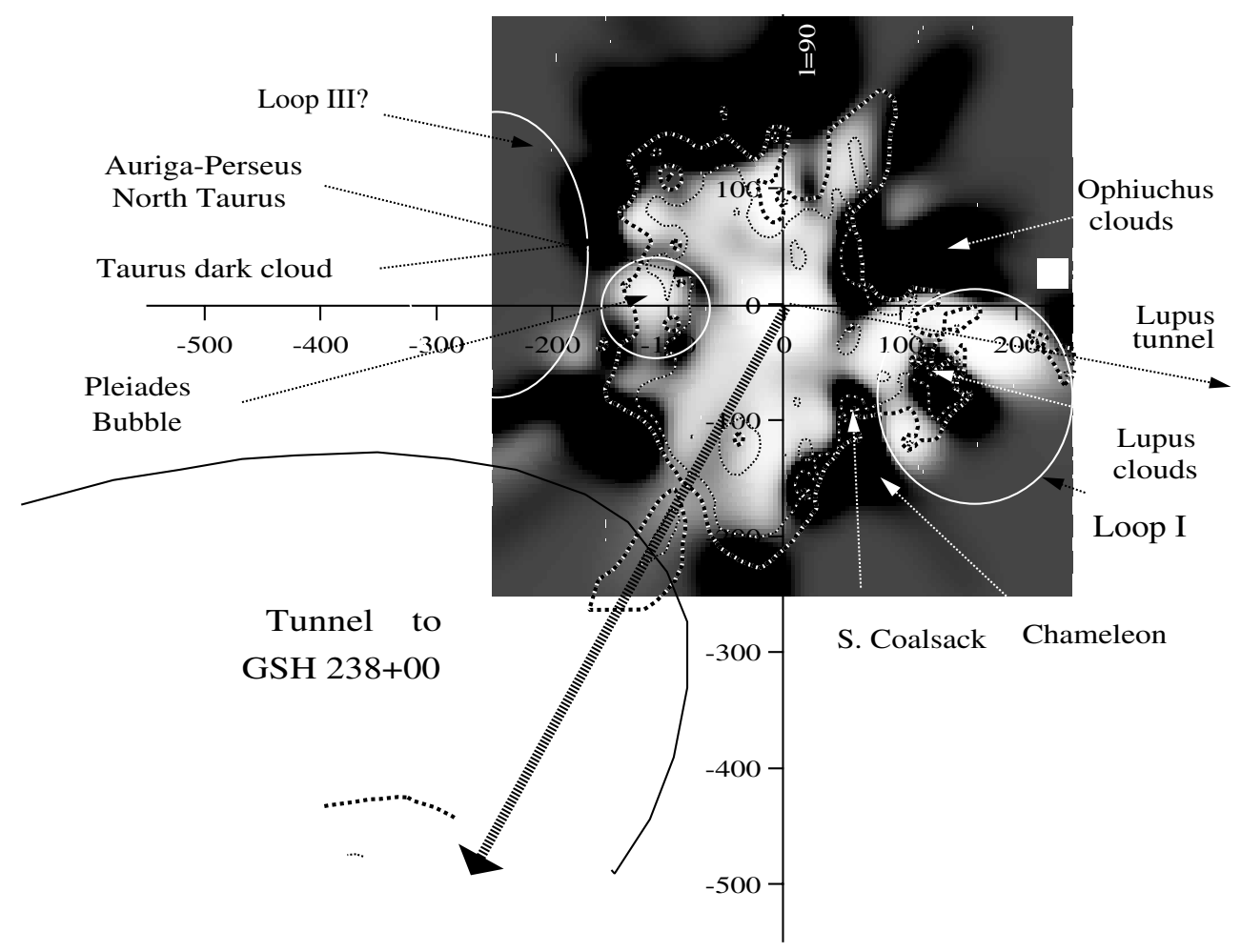

Fig. 9. NaI gas in the galactic plane and the connection to nearby shells.

closer, at less than $100 \mathrm{pc}$, between $l=70^{\circ}$ and $l=90^{\circ}$. More observations are certainly needed in this area, because from the present data set it is not clear whether or not the latter condensation is really embedded and isolated inside the LB.

In the third quadrant the data clearly show the extension to the low density LB cavity in the direction towards the stars $\epsilon$ and $\beta \mathrm{CMa}\left(l \sim 230^{\circ}\right.$ to a distance of $\sim 250 \mathrm{pc}$. $)$ This Canis Major cavity (Gry et al. 1985; Welsh 1991) is now revealed to extend further to at least $500 \mathrm{pc}$ (see Fig. 9) in a narrow "interstellar tunnel" or pathway that links the local cavity with that of the GSH $238+00+09$ supershell recently discovered by Heiles (1998), confirming the structure he has suggested.

It is clear that such physical links between these interstellar cavities strongly support the idea of networks of interstellar tunnels first fowarded nearly 30 years ago by Cox \& Smith (1974), in which expanding supernova-driven bubbles interact and merge to form large-scale interstellar cavities similar to the Heiles HI shells (Heiles 1979).

In Fig. 9 we sketch the positions of the major interstellar $\mathrm{HI}$ shells and superbubbles that are currently known to surround the LB region (Heiles 1998; Gahm 1994). As outlined in previous works (Welsh et al. 1994; Egger \& Aschenbach 1995), the morphology of the LB cavity seems to be defined by the intersections of several of these large surrounding interstellar structures such as the North Polar Spur (Radio Loop I) shell, the GSH238+00+O9 supershell, the Taurus-Perseus clouds and the Pleiades bubble (Lilienthal \& de Boer 1991), and possibly LoopIII. Although the actual sizes and boundaries of all these shells are presently not well defined, it seems clear from the shape of the neutral gas boundary to the LB that it appears to be being "squeezed" by these expanding supershells.

\section{The LISM viewed in the meridian plane - Fig. 5}

Due to the lack of availability of suitable target sight-lines in Paper I, the LB contour boundary was only plotted to a distance of $200 \mathrm{pc}$ in this galactic projection. Our new plot of Fig. 5 clearly shows the significant elongation of the low density LB cavity extending at least to a distance of $>250 \mathrm{pc}$ into the lower inner-halo regions of both galactic hemispheres. This interstellar feature has been termed the "Local Chimney" (LC) by Welsh et al. (1999) and the physical state of the gas in this low-density sight-line has been the focus of two recent interstellar studies by Crawford et al. (2002) and Welsh et al. (2002). As yet, no distinct and continuous neutral boundary to the ends of the LC has been found in either galactic hemisphere for distances $<400 \mathrm{pc}$, and Crawford et al. (2002) favor a picture of the inner galactic halo (in the southern hemisphere) in which a population of scattered, generally infalling, discrete diffuse clouds lie along the LC sight-line.

In Fig. 5 we now note the presence of only two major condensations of dense neutral gas (with a D2-line $E W>50 \mathrm{~m} \AA$ ) residing within the LB cavity, as opposed to the findings of Paper I in which several condensations were reported to lie within its neutral boundary. The complex of gas (presently defined by the NaI absorption levels observed towards the stars HD 14613 and HD 14670) at $\left(l=192^{\circ}, b=-67^{\circ}\right)$ can be confidently associated with the translucent cloud G192-67 at a distance of $\sim 110 \mathrm{pc}$ below the galactic plane (Grant \& Burrows 1999). Indeed, those stars have been used by these authors to locate the molecular gas and have been included in our database. This small dense cloud again illustrates the complementary nature of the two methods. The iso-contour boundary 
method provides the more precise location for the dense gas of G192-67, but the contours beyond the cloud (which are determined from distant targets with sight-lines angularly close to it, which may or may not intercept it), are badly defined, as can be seen for the $>50 \mathrm{~m} \AA$ contour beyond G192-67 which shape is very irregular. On the other hand, the density calculation locates the cloud slightly closer to the Sun (due to rejections of data points, as discussed in Sect. 4.3), but the cloud is cleary shown to be surrounded by an empty volume. As a confirmation exercise, we have removed all absorption components occuring at the velocity of the translucent cloud from all the targets with sight-lines in this direction. This results in a new calculation of the iso-contour which suppresses the previous irregularities and shows that there is no other dense gas detected in this region up to at least $200 \mathrm{pc}$, and that G192-67 is the perfect candidate for soft X-rays shadows.

The second dense condensation of gas which is clearly revealed is the HI shell in front of Taurus-Auriga (Lilienthal \& de Boer 1991), which has already been discussed in the previous section, and which this time is viewed from an observer located along the $(l, b)=(90,0)^{\circ}$ axis. Finally, towards the galactic center, the cut through the $3 \mathrm{D}$ distribution crosses the low density interstellar tunnel extending to $\sim 250 \mathrm{pc}$ in the direction of Sco-Cen as previously mentioned in Sect. 3.2.

Finally, contours in Fig. 5 also reveal the presence of three lower density condensations (of NaI D2-line $E W \sim 20 \mathrm{m \AA}$ ) within the LB boundary. The most prominant of these regions is the gas surrounding the star 2 Cet at $70 \mathrm{pc}$, which was discussed in Paper I. The density calculation does not produce any visible enhancement in this region, because the column density is small and other angular close lines-of-sight do conflict with this particular one. The other two lower density condensations are associated with (i) the chemically peculiar star HD 225206 at a distance of $244 \mathrm{pc}$ in the direction $\left(l=18^{\circ}, b=-79^{\circ}\right)$ and (ii) the rapidly rotating A2 star HD 72524 at a distance of $98 \mathrm{pc}$ in the direction $\left(l=186^{\circ}, b=+36^{\circ}\right)$. Although the last LOS may reveal an extension of the Auriga-Perseus shell, for these three objects a non-interstellar origin of the absorption is not precluded.

\section{The LISM viewed in the rotational plane - Fig. 6}

Figure 6 shows the projection perpendicular to that of the meridian plane shown in Fig. 5. Figure 6 contains many new target sight-lines and is the most changed of any of the projection plots originally shown in Paper I. The local cavity is now shown to be much less opened in the north in this plane, with only a narrow "chimney" towards the north galactic pole. This LC begins to narrow at a distance of $\sim 200 \mathrm{pc}$ above the plane. This confirms the pattern seen in the meridian plane, that the northern opening is tilted towards $l=180^{\circ}, b=+70^{\circ}$.

On the other hand, the LB cavity at highly negative galactic latitudes is seen to extend to at least $400 \mathrm{pc}$ into the halo, as was already seen in the meridian plane projection of Fig. 5.

There are two main dense condensations within the local cavity revealed by this plot. The first region lying at $\sim+35^{\circ}$ above the galactic plane at a distance of $\sim 80 \mathrm{pc}$ is in the sight-line to the two stars HD 156559 and HD 156338.
Wennmacher et al. (1992) have associated this particular interstellar cloud with the feature named LVC $88+36-2$, which has been shown to cast a shadow in the soft X-ray background (listed as S0862P383 in Snowden et al. 2000).

The contours reveal a second dense condensation of neutral gas lying within the LB cavity, seen at $\sim 150 \mathrm{pc}$ in the sight line $\left(l=285^{\circ}, b=-3^{\circ}\right)$ towards HD $81188(\kappa \mathrm{Vel})$ and HD 91465 . We note that the interstellar NaI D2-line is detected with a strength of $>100 \mathrm{~m} \AA$ and at a velocity of $V_{\text {helio }}=+7 \mathrm{~km} \mathrm{~s}^{-1}$ in both absorption spectra. However, this gas is probably physically linked to the cloud detected at a similar velocity towards HD 81157 which lies along the same sight-line but at a distance of only $85 \mathrm{pc}$. This means that there is a cloud with a very small angular extent closer than $85 \mathrm{pc}$, and that beyond the density falls off. The calculated density shows such a distribution, but has put the cloud at 60-70 pc, a distance which may be underestimated. Further work is needed to check whether this clump of gas is detected in radio or IR data.

Several less dense "clouds" of neutral gas (detected with a NaI D2-line equivalent width of $\sim 20 \mathrm{~m} \AA$ ) are also shown in Fig. 6. Of particular note is the gas detected towards the star 2 Cet at a distance of $\sim 70$ pc (see Paper I for a fuller discussion), and the gas associated with HD 192640 ( $d=41 \mathrm{pc})$ which may be circumstellar.

The extensions to the LB cavity at $\left(l=92^{\circ}, b=-33^{\circ}\right)$ reach out as far as $150 \mathrm{pc}$ to the star HD 217715 , and this feature can be considered as another of the lower neutral density interstellar tunnels that eminate from the local cavity in several galactic directions.

\subsection{Other vertical planes}

Figures 7 and 8 show the isocontours and densities in vertical planes crossing the galactic plane every 15 degrees longitude. For continuity we have kept the meridian and rotation plane in the series. Due to the smoothing length of $25 \mathrm{pc}$, the closest features can be followed from one plane to the other. When looking at those figures, it is important to have in mind that where there are no target stars, the density keeps the a priori value, i.e. the exponential distribution.

There are several cavities which appear in these intermediate planes which could not be seen in the main 3 planes. In particular, in the $45-225^{\circ}$ plane the Hercules shell (Lilienthal et al. 1992) is visible around $b=+45^{\circ}$, approaching as close as $70 \mathrm{pc}$ (see the discussion on MBM40). The cavity behind the shell is also clearly seen at about $150 \mathrm{pc}$. The $150-330^{\circ}$ and $165-345^{\circ}$ planes show more clearly the tunnels towards Loop I. The small cavity towards $l=90-120^{\circ}$ and $b=-35^{\circ}$ and extending to 200 pc may be associated with Loop II.

\subsection{Comparison with other data}

Our maps of the local distribution of the NaI ion (which samples gas with an ionization potential $<5.14 \mathrm{eV}$ ) clearly show the LB cavity to possess an extremely low density of cold and neutral gas. However, interstellar observations of the local ISM using ions of a higher ionization potential (such as CaII, MgII 
and FeII) have revealed the presence of many small, low density clouds (often referred to as "fluff"). The physical structure of some of these warm, ionized gas clouds lying within $100 \mathrm{pc}$ have been reviewed by Redfield \& Linsky (2002), but as yet no global mapping of either the kinematic or density structure of this ionized component of the LB gas has been achieved due to the small number of sight-lines thus far sampled. Thus, our present data is best compared with other tracers of the neutral component of the local ISM such as interstellar dust and neutral interstellar hydrogen (HI) gas.

The distribution of interstellar dust within $300 \mathrm{pc}$ has recently been discussed by Knude \& Høg (1999). Using Hipparcos distances and stellar colours from the Tycho catalogue, plots of the distribution of interstellar reddening revealed several interesting, apparently nearby features with colour excesses much larger than the sample average. In particular, an anomalous reddening value was found for the small region $\left(l=145^{\circ}, b=+65^{\circ}\right)$ that was tentatively associated with the IV Arch feature (Kuntz \& Danly 1996) possibly located at a distance as close as $50 \mathrm{pc}$. The nearest sight-line to this position for which we have NaI data is towards HD 104241 $\left(l=150^{\circ}, b=+70^{\circ}\right)$ with a distance of $175 \mathrm{pc}$. The low level of $\mathrm{NaI}$ seen towards this direction does not support the presence of dust as close as 50 pc. Additionally, Knude \& Høg (1998) have used the Hipparcos-Tycho data sets to derive distances for the nearest four southern star forming clouds in the Chamaeleon $(d=150 \mathrm{pc})$ and Lupus region $(d=100 \mathrm{pc})$. Our absorption maps of the local gas clearly place both of these star forming regions beyond the neutral boundary to the LB.

Vergerly et al. (2001) have presented 3D maps of the NaI and HI density distribution in the solar neighbourhood. The density of interstellar matter derived from $\mathrm{NaI}$ absorption data utilized the data set presented in Paper I and will thus not be discussed further. The density of matter derived for HI absorption used the data compilation of Garcia (1991) and Vergely et al. noticed that the size of the LB as revealed by HI was smaller than that derived from the $\mathrm{NaI}$ data, in the sense some clouds appear very clearly in the HI maps and not in the $\mathrm{NaI}$ maps. It was argued that since the first ionization state of $\mathrm{NaI}(5.3 \mathrm{eV})$ is below that of $\mathrm{HI}(13.6 \mathrm{eV})$, as the temperature of the interstellar gas rises $\mathrm{NaI}$ becomes ionized even though $\mathrm{HI}$ can remain predominantly neutral. These clouds should be detected by the CaII absorption.

Inspection of the absorption maps of HI presented in Fig. 10 of Vergely et al. (2001) show those regions of HI absorption with no counterpart in sodium. There is essentially one cloud at about $100 \mathrm{pc}$ towards the south pole direction, which may be associated with 2 Cet.

\section{Molecular clouds}

Several papers using absorption measurements have placed high latitude molecular clouds within the local hot bubble (e.g. Hobbs et al. 1986, 1988; Hearty et al. 2000). Most of them are the so-called MBM's, from Magnani et al. (1985). Indeed, our present database contains most of the data on sodium absorption used for this purpose. Such measurements are important, because knowledge of the distance and placement of molecular clouds with respect to the boundary of the LB is of great importance in the interpretation of the galactic distribution of soft X-ray background emission. In fact, if translucent or dark clouds are closeby and within the LB cavity, then these are the best candidates for X-ray shadowing measurements (Burrows \& Mendenhall 1991; Snowden et al. 2000). In addition, the location of these clouds with respect to the LB boundaries may also bring constraints on the LB history.

Those observing programs devoted to the localisation of the high latitude molecular clouds have made use of specific targets selected for their centering on radio or IR bright areas. On the contrary, our target stars are completely independently chosen, and for this reason we are not expecting to bring important new constraints.

Nonetheless, there are two ways our data may improve constraints on the distances. First, because our own lines-of-sight are randomly distributed, some of the new data may by chance complement those dedicated to distance estimates. Second, some of the searches have been done prior to Hipparcos results, and updating the stellar distances may change the final placement of the translucent cloud.

As discussed previously the most prominent isolated feature in our figures is the molecular cloud complex of G192-67 $\left(l=192.3^{\circ}, b=-67.9^{\circ}\right)$ at a distance of $110 \mathrm{pc}$. We have included in the database the target stars used by Grant $\&$ Burrows (1999), which allows us to locate the translucent cloud very precisely. The most interesting aspect about this cloud is that there is no dense gas beyond it up to at least $200 \mathrm{pc}$.

While an extensive comparison between $\mathrm{NaI}$ and radio data is beyond the scope of this paper, we have extracted from our list of stars those whith $E W(\mathrm{NaI}-\mathrm{D} 2)>100 \mathrm{~m} \AA$ and compared their directions with the directions of the 120 molecular clouds listed by Magnani et al. (1996). For each NaI target sight-line that is coincident with that of a known molecular cloud we have compared NaI velocities and cloud velocities to investigate whether or not a new constraint on the distance could be brought. The results are listed below (we follow the same order as in Magnani et al. 1996).

MBM3-4 $\left(l=131^{\circ}, b=-46^{\circ}\right)$ : The distance to this cloud was bracketed by Penprase (1993) and found to be 90-180 pc. One of the target stars of Penprase (1992) is at 153 pc (instead of $260 \mathrm{pc}$ ), and has a $\mathrm{NaI}$ absorption velocity within $2 \mathrm{~km} \mathrm{~s}^{-1}$ of the radio velocity. This locates the cloud closer than $170 \mathrm{pc}$, when taking into account the error on the parallax. This is a very small improvement with respect to the previous determination. However, we note that the sodium column towards this star is not very high, that no $\mathrm{CH}$ was detected, and that larger columns are found only towards much more distant stars. It is thus still possible that the molecular clump is more distant.

MBM 12: The distance to the MBM 11-12-13 complex (around $l=162^{\circ}, b=-35^{\circ}$ ) is a controversial subject. We observe that the four stars of Hobbs (1986) showing strong detections are located in the interval 90 to $150 \mathrm{pc}$. There is thus dense gas closer within $90 \mathrm{pc}$ from the Sun in this direction (the minimum distance is $60 \mathrm{pc}$ if one considers foreground stars within $1 \mathrm{deg}$ from the three stars showing the largest column, $90 \mathrm{pc}$ if one considers target stars within 
5 degrees). However, we note that $\mathrm{NaI}$ velocities are found to be between -1 and $+9 \mathrm{~km} \mathrm{~s}^{-1}$, while velocities observed at radio wavelengths are quoted by Magnani et al. (1996) to be around -5 and $-7 \mathrm{~km} \mathrm{~s}^{-1}$. Moreover, Luhman (2001) and Chauvin et al. (2002) have recently claimed that MBM12 is much more distant, at more than $200 \mathrm{pc}$, based on stellar fields properties. We suggest that there is an accidental coincidence between the direction of the molecular star-forming region and closer dense HI clouds at $90 \mathrm{pc}$.

MBM16: Hobbs et al. (1988) have located this cloud $(l=$ $172^{\circ}, b=-38^{\circ}$ ) between 60 and $95 \mathrm{pc}$. NaI and $\mathrm{CO}$ velocities are similar. From Hipparcos parallaxes the maximum distance is $118 \mathrm{pc}$ instead of $95 \mathrm{pc}$, while from foreground stars the minimum distance is about 90 pc. This is one of the clouds which is possibly at the boundary of the LB (see Fig. 4).

UT 3-7: The complex UT $3-7$ at $l=163$ to $168^{\circ}, b=-24$ to $-26.5^{\circ}$ is very likely closer than $100 \mathrm{pc}$, because all stars observed by White et al. (2001) and more distant than about 90-100 pc have prominent $\mathrm{NaI}$ lines at a heliocentric velocity of 15 and $17 \mathrm{~km} \mathrm{~s}^{-1}$, (or $6.5-8.5 \mathrm{~km} \mathrm{~s}^{-1} \mathrm{LSR}$ ), similar to the velocity of the molecular species. From the White et al. targets, and our additional targets, the complex should be located between 75 and $100 \mathrm{pc}$. This places it at the boundary of the LB (see Fig. 8), and in continuity with MBM16.

MBM18: Using Hipparcos parallaxes the distance to this cloud $\left(l=189^{\circ}, b=-36^{\circ}\right)$ is less than $165 \mathrm{pc}$ (including the parallax error) and larger than $65 \mathrm{pc}$. This is similar to the determination of Franco (1989) of 110-150 pc based on stellar photometry, and the cloud appears at closer distance than the 140-220 pc interval of Penprase (1993) from pre-Hipparcos distances. From the general density calculation, the favoured distance is of the order of $100 \mathrm{pc}$ and the cloud again is at the border of the LB, and in continuity of MBM16 and UT 3-7.

MBM 33-34-CB63-MBM 36-37 $\left(l=0-10^{\circ}, b=\right.$ $\left.+33-35^{\circ}\right)$ : Using our stars and the targets of Genova et al. (1997), we find $\mathrm{Na}$ lines at the same velocity as the radio data in the spectra of two stars closer at less than 108 and 125 pc (including errors). Foreground stars are missing and imply a minimum distance of about $50 \mathrm{pc}$ only. However, the general pattern favors a distance of about $100 \mathrm{pc}$, in agreement with Franco (1989) based on photometry, and again at the boundary of the LB.

MBM40 $\left(l=37.6^{\circ}, b=44.7^{\circ}\right)$ : This cloud is now found to be closer than the target star HD145146 (82 pc, upper limit $94 \mathrm{pc}$ ) in exactly the same direction, which shows a strong absorption line at $V(\mathrm{LSR})=0 \pm 1 \mathrm{~km} \mathrm{~s}^{-1}$, similar to the LSR velocity from radio data $(+2.5)$, and identical to the $\mathrm{NaI}$ and $\mathrm{CH}$ velocities from Penprase (1993). This is in agreement with the upper limit of Welty et al. (1989) and confirms the suggestion of Lilienthal et al. (1992) that MBM40 lies in front of the nearest arm of the Hercules shell, therefore producing an additional X-ray shadow. Updating the distances to the target stars of Lilienthal et al. we now locate more precisely the front of the Hercules shell at about $90 \mathrm{pc}$. With a minimum distance of about $50 \mathrm{pc}$ for MBM40 (and a favoured distance of about $80 \mathrm{pc}$ ), both features form the LB boundary between 30 and 70 degrees galactic longitude.
MBM41-44 (Draco clouds) and HI filament: For these objects at $\left(l=90^{\circ}, b=+38^{\circ}\right)$ the NaI velocities observed by Wenmacher et al. (1992) and others are different from the radio velocities (Lilienthal et al. 1991). While the molecular clouds are likely as distant as $200 \mathrm{pc}$, the HI dense filament observed by Wenmacher et al. (1992) is much closer. We find a new Hipparcos based maximum distance of $86 \mathrm{pc}$ (instead of $60 \mathrm{pc}$ ). This cloud again seems to be the closest dense cloud in this direction.

MBM54-55 $\left(l=3^{\circ}, b=-37^{\circ}\right)$ : In agreement with Welty et al. (1989), this cloud is closer than $110 \mathrm{pc}$, based on velocity comparisons. Foreground stars place it beyond $80 \mathrm{pc}$. It is located at the periphery of the LB.

G102-27: The distance to this cloud was not defined prior to our work. From velocity comparisons on one of our stars, HD 220599, its maximum distance is probably $155 \pm 20 \mathrm{pc}$.

Other high latitude condensations can be identified with high NaI columns, but distances are not better constrained from the present set of data.

The densest low latitude clouds have recently been located with precision from combined Hipparcos photometry and parallaxes, by Knude \& Høg (1998). We have indicated in Figs. 9 and 10 their resulting locations. They coincide very well with the dense regions mapped with NaI. In particular, the maps show clearly the continuity between the Coalsack and the Chameleon dark clouds.

\section{Conclusion}

We have presented new high spectral resolution observations of the interstellar NaI D-lines at $5890 \AA$ seen in absorption towards 311 stars located within $\sim 350$ pc of the Sun. Using these new data taken together with previous absorption measurements along another 694 sight-lines, we have constructed 3D maps of the galactic distribution of neutral sodium absorption towards a total of 1005 stars in the local interstellar medium.

These new data confirm many of the findings first presented in Paper I, but due to the factor of two increase in sight-lines presently sampled the placement of the neutral boundary to the LB is now known to an accuracy of $\sim \pm 20$ pc in many galactic directions. This neutral gas boundary surrounds the low density cavity of the Local Bubble from a distance of $\sim 60 \mathrm{pc}$ towards the galactic center direction, to hundreds of parsecs towards the northern and southern "chimneys", and inside the "tunnels" towards the surrounding loops. The "tunnels" to the Loop1 bubble and to the supershell GSH238+00+09 are the most visible. The properties of the LB boundaries may bring some clues about its history: apparently the LB is now in the process of being "squeezed" by surrounding expanding shells, which suggests it is much older and at lower pressure than the neighbouring shells. What about its elongation along an axis which is perpendicular to the Gould belt plane (Fig. 5)? Does it have some significance? Scenarios for the origin of the Gould Belt are various: impact of a huge external cloud with the galactic disk, highly energetic supernova(ae), or a gamma burst (see Perrot \& Grenier 2003). In the latter case, the LB could be the remnant of the central region of the burst, after full 


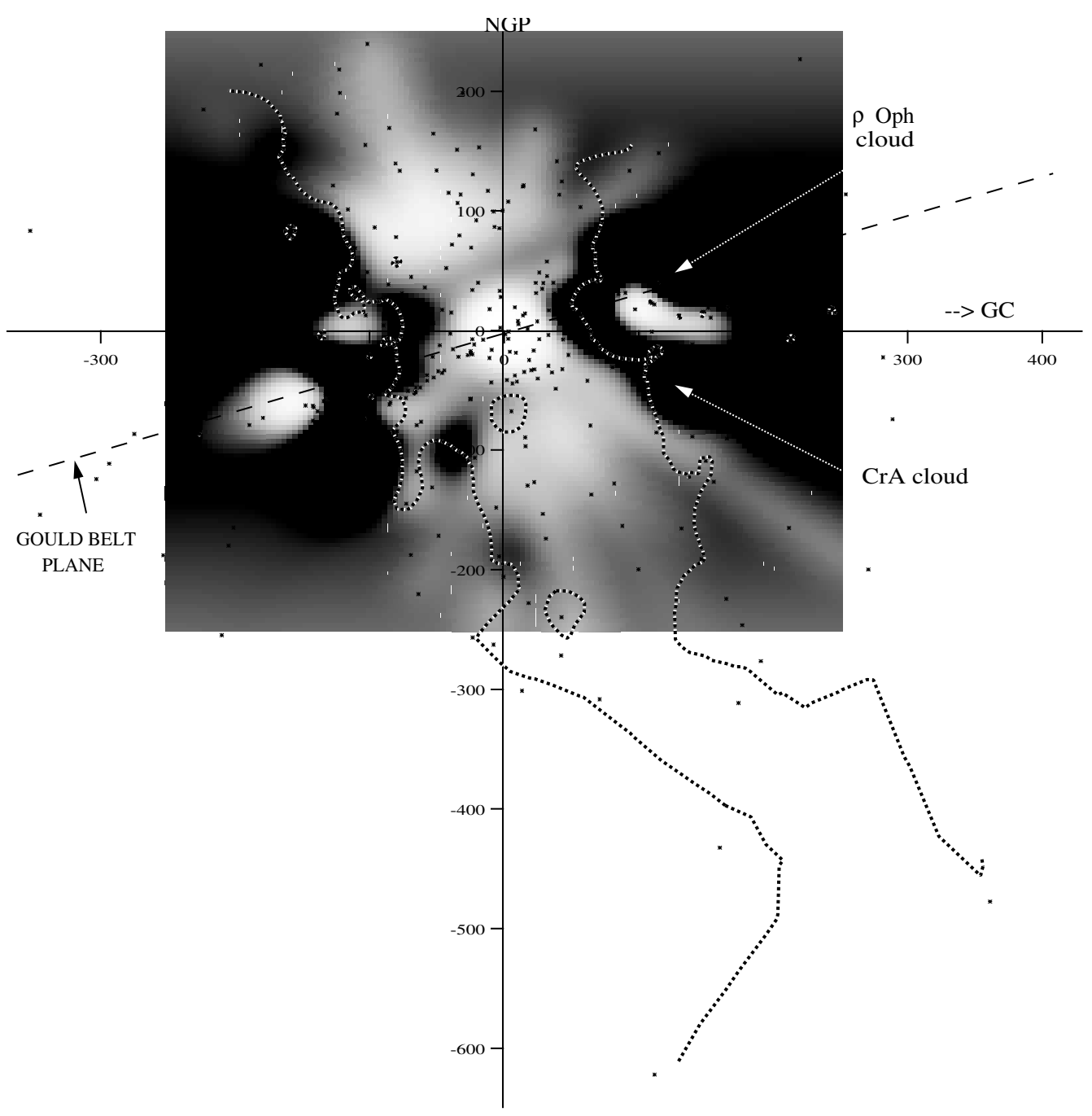

Fig. 10. NaI gas in the meridian plane. The most distant stars with negligible sodium are along an axis inclined by about 20 deg, i.e. perpendicular to the Gould belt plane.

ionization and sweeping of the gas, then slow recombination and squeezing by the successive generations of expanding shells associated to the belt. This speculative scenario could explain why the LB chimney opens towards more intermediate velocity clouds (IVC's) and high velocity clouds (HVC's) compared to other regions of the halo, if these clouds are formed from material ejected after the burst and falling back now onto the disk. It could also possibly explain why there is dust with no (or very small) gas counterpart under the IVC's at low altitude (Knude \& Høg 1999), if the gas has been expelled by a huge burst-generated pressure increase and has left dust behind it.

Regions of cold and dense interstellar gas are virtually nonexistant within the local cavity, with only a few small condensations having been definitely located within the LB boundary. Of these regions, the molecular cloud G192-67, which produces a remarkable $\mathrm{X}$-ray shadow, is the densest condensation which is clearly embedded within the LB.

On the other hand, several of the high latitude molecular clouds are found to be located at the periphery of the LB, i.e. they are at about the same distance as the nearest "wall" of dense gas detected in absorption. Having updated the distances to target stars from previous studies, and also added new targets, we are able to derive more accurate distances towards some of these clouds. We find that the nearest cloud is MBM40 at less than $82 \pm 12 \mathrm{pc}$. We are also able to confirm that this cloud is in front of the HI gas, as suggested by Lilienthal et al. (1992) and this explains its shadowing effect on the soft Xray background. The HI gas is found to be located at $\sim 90 \mathrm{pc}$. Further work is needed to measure the columns of HI gas in front of or beyond those clouds.

This work aims at providing a global frame for the interpretation of emission data, from radio and IR to EUV (CHIPS) and soft X-rays, as well as of soft X-ray shadows. For these purposes it needs to be complemented by the kinematical study of the NaI gas, from absorption line velocities (not used here) and by a mapping of the warm, diffuse, CaII bearing gas. This will be the subject of forthcoming studies.

Acknowledgements. We wish to thank the staffs of (by increasing west longitude) the Observatoire de Haute Provence (France), the Mount Stromlo Observatory (Australia), the Lick Observatory (California), the Kitt Peak National Observatory (Arizona), and the 
European Southern Observatory (Chile), for their great help in performing all these observations. DS and BYW acknowledge funding support through Dr. Oswald Siegmund and the NASA FUSE Guest Investigator program. The NSF did not consider this research worthy of their financial support.

R.L. acknowledges funding by the CNRS programs (Grands Telescopes Étrangers, PCMI) and the France-Australie cooperation program, and Sandra Werly for her help for a part of the profile fitting.

We also especially thank our referee who has performed an extremely careful reading of the paper and whose numerous suggestions have improved its clarity.

\section{References}

Berghöfer, T. W., \& Breitschwerdt, D. 2002, A\&A, 390, 299

Bertin, P., Vidal-Madjar, A., Lallement, R., Ferlet, R., \& Lemoine, M. 1995, A\&A, 302, 889

Breitschwerdt, D., Freyberg, M. J., \& Egger, R. 2000, A\&A, 361, 303

Breitschwerdt, D. 2001, Ap\&SS, 276, 163

Burrows, D., \& Mendenhall, J. 1991, Nature, 351, 629

Cha, A., Sahu, M., Moos, H. W., \& Blaauw, A. 2000, ApJS, 129, 281

Chauvin, G., Ménard, F., Fusco, T., et al. 2002, A\&A, 394, 949

Cox, D., \& Smith, B. 1974, ApJ, 189, L105

Cox, D. P. 1998, Lecture Notes in Physics (Berlin: Springer Verlag), 506, 121

Cravens, T. E. 2000, ApJ, 532, L153

Crawford, I. 1990, MNRAS, 243, 593

Crawford, I. 2000, MNRAS, 317,996

Crawford, I. 2001, MNRAS, 327, 841

Crawford, I., Lallement, R., Price, R. J., et al. 2002, MNRAS, 337, 720

Crutcher, R., \& Lien, D. 1984, in Local Interstellar Medium, ed. Y. Kondo, F. Bruhweiler, \& B. Savage (NASA CP-2345), IAU Colloq., 81, 117

Diplas, A., \& Savage, B. D. 1994, ApJS, 93, 211

Egger, R. J., \& Aschenbach, B. 1995, A\&A, 294, L25

ESA 1997, The Hipparcos and Tycho Catalogues, ESA SP-1200

Ferlet, R., Vidal-Madjar, A., \& Gry, C. 1985, ApJ, 298, 838

Franco, G. A. P. 1989, A\&A, 223, 313

Franco, G. 2000, MNRAS, 315, 611

Frisch, P., Sembach, K., \& York, D. 1990, ApJ, 364, 540

Fruscione, A., Hawkins, I., Jelinsky, P., \& Wiercigroch, A. 1994, ApJS, 94, 127

Gahm, G. 1994, Balt. Astr., 3, 85

Garcia, B. 1991, A\&AS, 89, 469

Genova, R., Beckman, J. E., Bowyer, S., \& Spicer, T. 1997, ApJ, 484, 761

Genova, R., \& Beckman, J. 2003, ApJSS, in press

Grant, C., \& Burrows, D. 1999, ApJ, 516, 243

Gry, C., York, D. G., \& Vidal-Madjar, A. 1985, ApJ, 296, 593

Gry, C., \& Jenkins, E. B. 2001, A\&A, 367, 617

Hearty, T., Fernandez, M., Alcala, J., Covino, E., \& Neuhauser, R. 2000, A\&A, 357, 681

Heiles, C. 1979, ApJ, 229, 533

Heiles, C. 1998, ApJ, 498, 689

Hobbs, L. M. 1978, ApJ, 222, 491

Hobbs, L. M., Blitz, L., \& Magnani, L. 1986, ApJ, 306, L109

Hobbs, L. M., Penprase, B., Welty, D., Blitz, L., \& Magnani, L. 1988, ApJ, 327, 356

Holberg, J., Bruhweiler, F., Barstow, M., \& Dobbie, P. 1999, ApJ, 517 , 841

Hurwitz, M., \& Sholl, M. 1999, BAAS, 195, 8806
Jenkins, E. B., Oegerle, W. R., Gry, C., et al. 2000, ApJ, 538, L81

Jenkins, E. B. 2002, ApJ, 580, 938

Kamp, I., \& Paunzen, E. 2002, MNRAS, 335, L45

Kemp, S., Bates, B., Beckmar, J. E., et al. 2002, MNRAS, 333, 561

Knude, J., \& Høg, E. 1998, A\&A, 338, 897

Knude, J., \& Høg, E. 1999, A\&A, 341, 451

Kuntz, K. D., \& Danly, L. 1996, ApJ, 457, 703

Lallement, R., Vidal-Madjar, A., \& Ferlet, R. 1986, A\&A, 168, 225

Lallement, R., Bertin, P., Chassefiere, E., \& Scott, N. 1993, A\&A, 271, 734

Lallement, R., \& Ferlet, R. 1997, A\&A, 324, 1105

Lallement, R. 1998, Lecture Notes in Physics (Berlin: Springer Verlag), 506, 19

Lilienthal, D., \& de Boer, K. 1991, A\&AS, 87, 471

Lilienthal, D., Hirth, W., Mebold, U., \& de Boer, K. 1992, A\&A, 255, 323

Luhman, K. L. 2001, ApJ, 560, 287

Magnani, L., Hartmann, D., \& Speck, B. G. 1996, ApJS, 106, 447

Maiz-Apellaniz, J. 1999, ApJ, 560, L83

Mebold, U., Kerp, J., \& Kalberla, P. M. W. 1998, Lecture Notes in Physics (Berlin: Springer Verlag), 506, 199

Oegerle, W., Jenkins, E. B., Shelton, R. L., Bowen, D. V., \& FUSE Team 2000, BAAS, 197.07

Paunzen, E., Duffee, B., Heiter, U., Kuschnig, R., \& Weiss, W. 2001, A\&A, 373, 625

Perrot, C., \& Grenier, I. 2003, A\&A, 404, 519

Penprase, B. 1993, ApJS, 88, 433

Redfield, S., \& Linsky, J. 2002, ApJSS, 139, 439

Robertson, I., Cravens, T., Snowden, S., \& Linde, T. 2001, Sp. Sci. Rev., 97, 401

Sfeir, D., Lallement, R., Crifo, F., \& Welsh, B. Y. 1999, A\&A, 346, 785 (Paper I)

Slavin, J. 1989, ApJ, 346, 718

Snowden, S., Egger, R., Finkbeiner, D. P., Freyberg, M. J., \& Plueinsky, P. P. 1998, ApJ, 493, 715

Snowden, S., Freyberg, M., Kuntz, K., \& Sanders, W. 2000, ApJS, 128,171

Stokes, G. 1978, ApJS, 36, 115

Vallerga, J. V., Vedder, P. W., Craig, N., \& Welsh, B. Y. 1993, ApJ, 411, 729

Vergely, J.-L., Freire Ferrero, R., Siebert, A., \& Valette, B. 2001, A\&A, 366, 1016

Vidal-Madjar, A., Lemoine, M., Ferlet, R., et al. 1998, A\&A, 338, 694

Welsh, B. Y. 1991, ApJ, 373, 556

Welsh, B. Y., Craig, N., Vedder, P., \& Vallerga, J. 1994, ApJ, 437, 638

Welsh, B. Y., Sasseen, T., Craig, N., Jelinsky, S., \& Albert, C. 1997, ApJS, 112, 507

Welsh, B. Y., Sfeir, D., Sirk, M., \& Lallement, R. 1999, A\&A, 352, 308

Welsh, B. Y., Sallmen, S., Sfeir, D., Shelton, R., \& Lallement, R. 2002, A\&A, 394, 691

Welsh, B. Y., Sallmen, S., Sfeir, D., \& Lallement, R. 2002, A\&A, 391, 705

Welsh, B. Y., Sallmen, S., Jelinsky, S., \& Lallement, R. 2003, A\&A (submitted)

Welty, D., Hobbs. L., Penprase, B., \& Blitz, L. 1989, ApJ, 346, 232

Welty, D. E., Hobbs, L. M., \& Kulkarni, V. 1994, ApJ, 436, 152

Welty, D., Morton, D., \& Hobbs, L. M. 1996, ApJS, 106, 533

Wennmacher, A., Lilienthal, D., \& Herbstmeier, U. 1992, A\&A, 261, L9

White, R., Allen, C., Forrester, W., et al. 2001, ApJ, 132, 253

Wolff, B., Koester, D., \& Lallement, R. 1999, A\&A, 346, 969 